\title{
Expression signature, prognosis value and immune characteristics of cathepsin $F$ in non-small cell lung cancer identified by bioinformatics assessment
}

Liyuan Song ${ }^{1 \dagger}$, Xianhui Wang ${ }^{2 \dagger}$, Wang Cheng ${ }^{1}$, Yi Wu ${ }^{1,3}$, Min Liư ${ }^{3}$, Ruizi Liu ${ }^{4}$, Shenyi Zhang ${ }^{1}$, Hong Xia ${ }^{1}$, Hao Liu ${ }^{1}$, Xuejiao Tai ${ }^{5}$, Huzi Zhao ${ }^{5}$, Xihua $\mathrm{Li}^{1^{*}}$ and Fuyun $\mathrm{Ji}^{1,6^{*}}$

\begin{abstract}
Background: In recent years, immunotherapies and targeted therapies contribute to population-level improvement in NSCLC cancer-specific survival, however, the two novel therapeutic options have mainly benefit patients containing mutated driven genes. Thus, to explore other potential genes related with immunity or targeted therapies may provide novel options to improve survival of lung cancer patients without mutated driven genes. CTSF is unique in human cysteine proteinases. Presently, CTSF has been detected in several cell lines of lung cancer, but its role in progression and prognosis of lung cancer remains unclear.

Methods: CTSF expression and clinical datasets of lung cancer patients were obtained from GTEx, TIMER, CCLE, THPA, and TCGA, respectively. Association of CTSF expression with clinicopathological parameters and prognosis of lung cancer patients was analyzed using UALCAN and Kaplan-Meier Plotter, respectively. LinkedOmics were used to analyze correlation between CTSF and CTSF co-expressed genes. Protein-protein interaction and gene-gene interaction were analyzed using STRING and GeneMANIA, respectively. Association of CTSF with molecular markers of immune cells and immunomodulators was analyzed with Immunedeconv and TISIDB, respectively.

Results: CTSF expression was currently only available for patients with NSCLC. Compared to normal tissues, CTSF was downregulated in NSCLC samples and high expressed CTSF was correlated with favorable prognosis of NSCLC. Additionally, CTSF expression was correlated with that of immune cell molecular markers and immunomodulators both in LUAD and LUSC. Noticeably, high expression of CTSF-related CTLA-4 was found to be associated with better OS of LUAD patients. Increased expression of CTSF-related LAG-3 was related with poor prognosis of LUAD patients while there was no association between CTSF-related PD-1/PD-L1 and prognosis of LUAD patients. Moreover, increased expression of CTSF-related CD27 was related with poor prognosis of LUAD patients while favorable prognosis of LUSC patients.
\end{abstract}

Conclusions: CTSF might play an anti-tumor effect via regulating immune response of NSCLC.

\footnotetext{
*Correspondence: Ixihua2018@hbmu.edu.cn; jifuyun@263.net;

jfuyun2018@hbmu.edu.cn

tLiyuan Song and Xianhui Wang have contributed equally to this work

${ }^{1}$ Department of Medical Biology, School of Basic Medical Science, Hubei

University of Medicine, Shiyan 442000, Hubei Province, China

Full list of author information is available at the end of the article
}

(C) The Author(s) 2021. Open Access This article is licensed under a Creative Commons Attribution 4.0 International License, which permits use, sharing, adaptation, distribution and reproduction in any medium or format, as long as you give appropriate credit to the original author(s) and the source, provide a link to the Creative Commons licence, and indicate if changes were made. The images or other third party material in this article are included in the article's Creative Commons licence, unless indicated otherwise in a credit line to the material. If material is not included in the article's Creative Commons licence and your intended use is not permitted by statutory regulation or exceeds the permitted use, you will need to obtain permission directly from the copyright holder. To view a copy of this licence, visit http://creativecommons.org/licenses/by/4.0/. The Creative Commons Public Domain Dedication waiver (http://creativeco mmons.org/publicdomain/zero/1.0/) applies to the data made available in this article, unless otherwise stated in a credit line to the data. 
Keywords: CTSF, NSCLC, Prognosis, Immune response, Immunotherapy

\section{Background}

Lung cancer is the most frequently occurring cancer and the leading cause of cancer death worldwide with an approximated 2.2 million new cancer cases and 1.8 million deaths in 2020 [1]. It is estimated that number of lung cancer deaths will increase to 3 million in 2035 [2, 3]. Non-small cell lung cancer (NSCLC) and small cell lung cancer (SCLC) are the two major histologic subtypes of lung cancer, and the former accounts for about $85 \%$ of all lung cancer cases and are mainly composed of lung adenocarcinoma (LUAD) and lung squamous cell carcinoma (LUSC). Prognosis for lung cancer is very poor. Five-year survival rates of lung cancer vary from $4-17 \%$ depending on subtypes and stage at the time of its diagnosis [4]. In recent years, immunotherapies and targeted therapies contribute to population-level improvement in NSCLC cancer-specific survival, however, the two novel therapeutic options have been mainly beneficial for those who contain the mutated driven genes, such as genes encoding PD-1/PD-L1 [5], anaplastic lymphoma kinase (ALK) [6], or epidermal growth factor receptor (EGFR) [7]. Thus, to explore other potential genes related with immunity or targeted therapies may provide novel options to improve survival of lung cancer patients who are not suitable for the current immunotherapies or targeted therapies.

Cysteine proteinases are a widespread group of enzymes that catalyze hydrolysis of many different proteins and play a major role in intracellular protein degradation and turnover $[8,9]$. These proteolytic enzymes can be subdivided into more than 20 different families, and papain family is the largest one [10]. Human cysteine proteinases of papain family are main component of lysosomal protein hydrolysis system and contain 15 different family members, including cathepsin A-H, K, L, O, S, V, $\mathrm{W}$, and Z [11]. These cathepsins (CTSs) contain a series of conserved features in their active site and are synthesized as preproenzymes, which are processed to corresponding proenzymes and targeted to lysosomes by mannose 6-phosphate signal attached to them [12]. Among these cysteine proteinases, lysosomal CTSB, CTSD, CTSL, and CTSS have been shown to be involved in tumor malignant progression and are potential targets of antitumor therapy [13-16]. Cloned from a human prostate cDNA library and unique in cathepsin because of having an extended $\mathrm{N}$-terminal anterior region containing a cystatin domain, CTSF gene encodes a polypeptide of 484 amino acids with the same domain organization as other cysteine proteinases. The wide expression of CTSF in human tissues suggests that CTSF may be involved in protein catabolism [12]. Additionally, CTSF likely plays a regulatory role in processing invariant chain which is associated with major histocompatibility complex (MHC) class II $[17,18]$. In fish, CTSF appears to take part in innate immune responses [19]. Presently, CTSF has been detected in some human cancer cell lines (such as HL-60, HeLa, K-562, MOLT-4, Raji, SW480, A549 and G361) [12], however, its role in progression and prognosis of tumors is still unclear. In the study, CTSF expression in NSCLC were analyzed using the public databases online available. The bioinformatics assessment revealed that CTSF may play anticancer effect in NSCLC by regulating immune responses.

\section{Materials and methods \\ CTSF expression in human cancers and cancer cell lines}

CTSF expression in human normal tissues and Pancancerous tumors were obtained from Genotype-tissue expression (GTEx) and Tumor Immune Estimation Resource (TIMER1.0), respectively. GTEx provides RNAseq data from 53 normal tissues across nearly 1000 people (http://xena.ucsc.edu/). TIMER is a comprehensive resource for systematical analysis of immune infiltrates across diverse cancer types (https://cistrome.shinyapps. io/timer/). CTSF expression in human normal tissues and Pan-cancers were analyzed using web browser-basis tools (https://gtexportal.org/home/gene/ENSG000001 74080 and https://cistrome.shinyapps.io/timer/, respectively). Expression of CTSF in human cancer cell lines was downloaded from Cancer Cell Line Encyclopedia (CCLE) (https://www.betastasis.com/tissues/cancer cell_line_encyclopedia/gene_expression_barplot/).

\section{Association of CTSF expression with prognosis of NSCLC} Expression of CTSF in LUAD and LUSC was derived from The Cancer Genome Atlas (TCGA) dataset (https://www.cancer.gov/about-nci/organization/ccg/ research/structural-genomics/tcga). TCGA has generated large amounts of Next-generation sequencing (NGS) data with a landscape of more than 11,000 tumors across 33 cancer types until 2018. Unpaired Wilcoxon test was used to evaluate distributions of CTSF expression in unpaired normal and tumor tissues of TCGA-LUAD and TCGA-LUSC cohorts, and paired Wilcoxon test was used to analyze CTSF expression between paired tumors and corresponding adjacent normal tissues of TCGA-LUAD and TCGA-LUSC cohorts. UALCAN was used to explore association of 
CTSF expression with clinicopathological parameters of LUAD and LUSC patients, including gender, age, smoking habits, stage, metastasis, TP53 mutation statuses and race (http://ualcan.path.uab.edu/cgi-bin/ TCGAExResultNew2.pl? genen am $=$ CTSF\&ctype $=$ LUAD, and http://ualcan.path.uab.edu/cgi-bin/TCGAE $x$ ResultNew2.pl? genen $a m=C T S F \& c t y p e=L U S C$, respectively). UALCAN allows analysis of relative expression of a query gene(s) across tumor and normal samples, as well as in various tumor sub-groups based on individual cancer stages, tumor grade or other clinicopathological features. One-way ANOVA was used to perform a comparison for continuous variables among groups $\geq 3$ on web browser-basis analysis. $P<0.05$ was considered statistically significant [20]. Immunohistochemistry (IHC) staining and subcellular localization of CTSF were derived from The Human Protein Atlas (THPA) dataset (https://www.proteinatlas.org/ENSG0 0000174080-CTSF/pathology/lung+cancer). THPA integrates RNA and protein expression data corresponding to $\sim 80 \%$ of human protein-coding genes with access to the primary data for both RNA and protein analysis on an individual gene level. Currently, CTSF expression in GTEx, TCGA and THPA databases are only available for LUAD and LUSC.

Kaplan-Meier Plotter was used to analyze CTSF expression with prognosis of NSCLC patients (http:// $\mathrm{kmplot} . \mathrm{com} /$ analysis/index.php? $\mathrm{p}=$ service\&cancer $=$ lung). Kaplan-Meier plotter can assess effect of 54,675 genes on survival of cancer patients with 10,461 cancer samples. Prognostic parameters included overall survival (OS), first-progression survival (FPS), and post-progression survival (PPS). FPS refers to the duration of disease progression from first-line treatment to first progression. PPS refers to the duration of disease progression from first-line treatment to death. Since post-progression therapy influences OS, PPS is of interest as a determinant of OS and considered as the difference between median OS and median progression-free survival (PFS) or time to tumor progression [21, 22]. Patients with LUAD and LUSC were divided into two subgroups based on median expression of CTSF (high vs. low expression), respectively. Survival diagram, Hazard ratio (HR) with 95\% confidence intervals (95\% CIs) and logrank $P$ value were calculated and plotted in R using the "survplot" function of the "survival" Bioconductor package as described [23].

Additionally, TCGA-LUAD and TCGA-LUSC database were used to analyze correlations of CTSF expression with tumor mutational burden (TMB) and microsatellite instability (MSI) in LUAD and LUSC, respectively. Spearman's correlation analysis was used to describe the correlation between quantitative variables without a normal distribution (https://www.aclbi.com/static/index.html\#/ tcga). A $p$ value of less than 0.05 was considered statistically significant.

\section{Genes co-expressed with CTSF in NSCLC}

LinkFinder module and LinkInterpreter module of LinkedOmics were used to analyze correlation of CTSF with other genes co-expressed with CTSF in LUAD and LUSC, respectively. LinkedOmics database is a Webbased platform for analyzing 32 TCGA cancer-associated multi-dimensional datasets (http://www.linkedomics. org/admin.php) [24]. All results were graphically presented in volcano plots, heat maps or scatter plots. Coexpressed genes with CTSF were categorized using Gene Ontology (GO) and Kyoto Encyclopedia of Genes and Genomes (KEGG) enrichment analysis (http://www.linke domics.org/admin.php).

Moreover, protein-protein interaction (PPI) network and gene-gene interaction network for CTSF were constructed using STRING and GeneMANIA, respectively. STRING is a flexible and user-friendly database of known and predicted PPIs, covering 24,584,628 proteins from 5,090 organisms currently (https://www.string-db.org/ cgi/network? taskId=bbIwHMDa6Z9y\&sessionId= bISFiNqXFgX5). Interactions in STRING include direct (physical) and indirect (functional) associations, stemming from computational prediction, knowledge transfer between organisms, and interactions aggregated from other (primary) databases. In the study, PPI network was constructed by setting medium confidence at 0.400 . GeneMANIA generates a list of genes with similar functions to the query gene and constructs an interactive functional-association network using a very large set of functional association data (http://genemania. org/search/homo-sapiens/CTSF). Association data include protein and genetic interactions, pathways, coexpression, co-localization and protein domain similarity. GeneMANIA can also be used to find new members of a pathway or complex, additional genes missed in the query input list or new genes with a specific function, such as protein kinases. In the present study, GeneMANIA was used to construct a gene-gene interaction network for CTSF to evaluate the potential functions of these genes. Each node represents a gene. The node color represents the possible functions of each gene.

\section{CTSF and molecular markers of immune cells in NSCLC}

Association of CTSF with molecular markers of immune cells between NSCLC and normal tissues was analyzed with Immunedeconv, an $\mathrm{R}$ package which integrates six state-of-the-art algorithms, including TIMER, xCell, MCP-counter, CIBERSORT, EPIC and quanTIseq (https://www.aclbi.com/static/index.html\#/immunoassay). TIMER was utilized to analyze correlation of copy 
numbers of CTSF gene with infiltration levels of immune cells using a two-sided Wilcoxon rank-sum test.

CTSF-related immunomodulators were analyzed with TISIDB to elucidate tumor-immune system interactions (http://cis.hku.hk/TISIDB/browse.php?gene=CTSF). TISIDB is a website for gene- and tumor-immune interaction, built based on data collected and integrated from following resources: PubMed database, high-throughput screening data investigating the responses of tumor cells to $\mathrm{T}$ cytotoxic cells, exome and RNA sequencing data of patients receiving immunotherapy, TCGA, and other public databases. Immunoinhibitors and immunostimulators that were significantly correlated with CTSF expression were chosen based on $p<0.05$ (Spearman correlation test). Association of immunomodulators with prognosis of LUAD and LUSC patients was analyzed using Kaplan-Meier plotter as described above.

\section{Results \\ CTSF was downregulated significantly in most of human cancer tissues}

CTSF was detected in almost all of human normal tissues. Relatively, higher expressed CTSF was observed in Artery-Aorta, Brain (cerebellar hemisphere and cerebellum), Cervix (ectocervix and endocervix), Fallopian tube, Nerve (tibial), ovary, Testis and Uterus (GTEx dataset, TPM $>200$, Fig. 1A). Exploration of CTSF expression in pan-cancers using TIMER revealed that compared to normal tissues, CTSF was downregulated significantly in most of cancer tissues, such as Bladder urothelial carcinoma (BLCA), Breast invasive carcinoma (BRCA), Cervical squamous cell carcinoma and endocervical adenocarcinoma (CESC), Cholangio carcinoma (COAD), Esophageal carcinoma (ESCA), Glioblastoma multiforme (GBM), Kidney chromophobe (KICH), Kidney renal clear cell carcinoma (KIRC), Kidney renal papillary cell carcinoma (KIRP), Lung adenocarcinoma (LUAD), Lung squamous cell carcinoma (LUSC), Rectum adenocarcinoma (READ), Stomach adenocarcinoma (STAD), Thyroid carcinoma (THCA), and Uterine corpus endometrial carcinoma (UCEC) (Fig. 1B). CTSF was detected in many human cancer cell lines including lung cancer (CCLE dataset, Additional file 1: Table S1).

\section{High CTSF expression was correlated with favorable prognosis of NSCLC}

As shown in Fig. 1C, unpaired Wilcoxon test demonstrated that CTSF expression was markedly downregulated both in LUAD (left) and LUSC (right) tissues compared to that of normal tissues. Paired Wilcoxon test revealed that CTSF expression was higher in some tumor tissues while lower in the other tumor tissues compared with that of the corresponding paracancerous tissues (Fig. 1D; left: LUAD; right: LUSC). Interestingly, IHC staining of CTSF showed that CTSF was not detected in alveolar cells while medium staining of CTSF was observed in macrophage cells in normal lung tissues (Fig. 1E). Moreover, stronger IHC staining of CTSF was detected in infiltrating immune cells such as macrophage cells than that of cancerous cells both in LUAD and LUSC tissues (Fig. 1E). As shown in Fig. 1F, CTSF was localized to plasma membrane, cytosol, and vesicles in tumor cells.

Analysis of CTSF expression with clinical characteristics of LUAD showed that CTSF expression was significantly associated with gender, age, tumor stage, lymph node metastasis, smoking habits, histological subtypes, TP53-muation status, and race, respectively. CTSF expression decreased with stage, metastasis and smoking in LUAD (Fig. 2A). Similar findings were obtained in LUSC (Fig. 2B). Kaplan-Meier survival analysis demonstrated that high CTSF expression was correlated with favorable prognosis of LUAD (OS: $\mathrm{HR}=0.46$, $p=5.7 \mathrm{e}-10$; FS: $\mathrm{HR}=0.54, p=1.3 \mathrm{e}-04$ ) (Fig. $2 \mathrm{C}$ ). In LUSC, high expressed CTSF was found to be significantly associated with better FS (HR $=0.56, p=0.025)$ (Fig. 2D).

Analysis of CTSF expression with TMB and MSI demonstrated that CTSF expression was negatively related with MSI $(p=0.002)$, while no significant association was found between CTSF expression and TMB in LUAD (Fig. 2E). There was no significant association between CTSF expression and TMB or MSI in LUSC (Fig. 2F).

\section{CTSF might play an important role in antigen presentation for immune response of NSCLC}

To better understand biological implications of CTSF in NSCLC, "LinkFinder" module in LinkedOmics was applied to explore genes co-expressed with CTSF. As plotted in Additional file 2: Fig. S1A, expression of 4,452 genes (red dots) was positively correlated with that of CTSF, while expression of 2,041 genes (green dots) was negatively correlated with that of CTSF in LUAD (FDR < 0.01). In LUSC, expression of 5,019 genes (red dots) was positively correlated with CTSF expression, while expression of 1,820 genes (green dots) was negatively correlated with CTSF expression (FDR $<0.01$, Additional file 2: Fig. S1B). Heatmaps of the top 50 genes positively or negatively associated with CTSF in LUAD and LUSC were shown in Fig. 3A and B, respectively. GO term annotation showed that molecular function of genes co-expressed positively with CTSF in LUAD were mainly involved in collagen trimer, extracellular matrix, transport complex, Golgi limen, and endoplasmic reticulum lumen. In contrast, molecular function of genes coexpressed negatively with CTSF were mainly involved in chromosomal region, condensed chromosome, preribosome, spindle, replication fork and others (GO-Molecular 


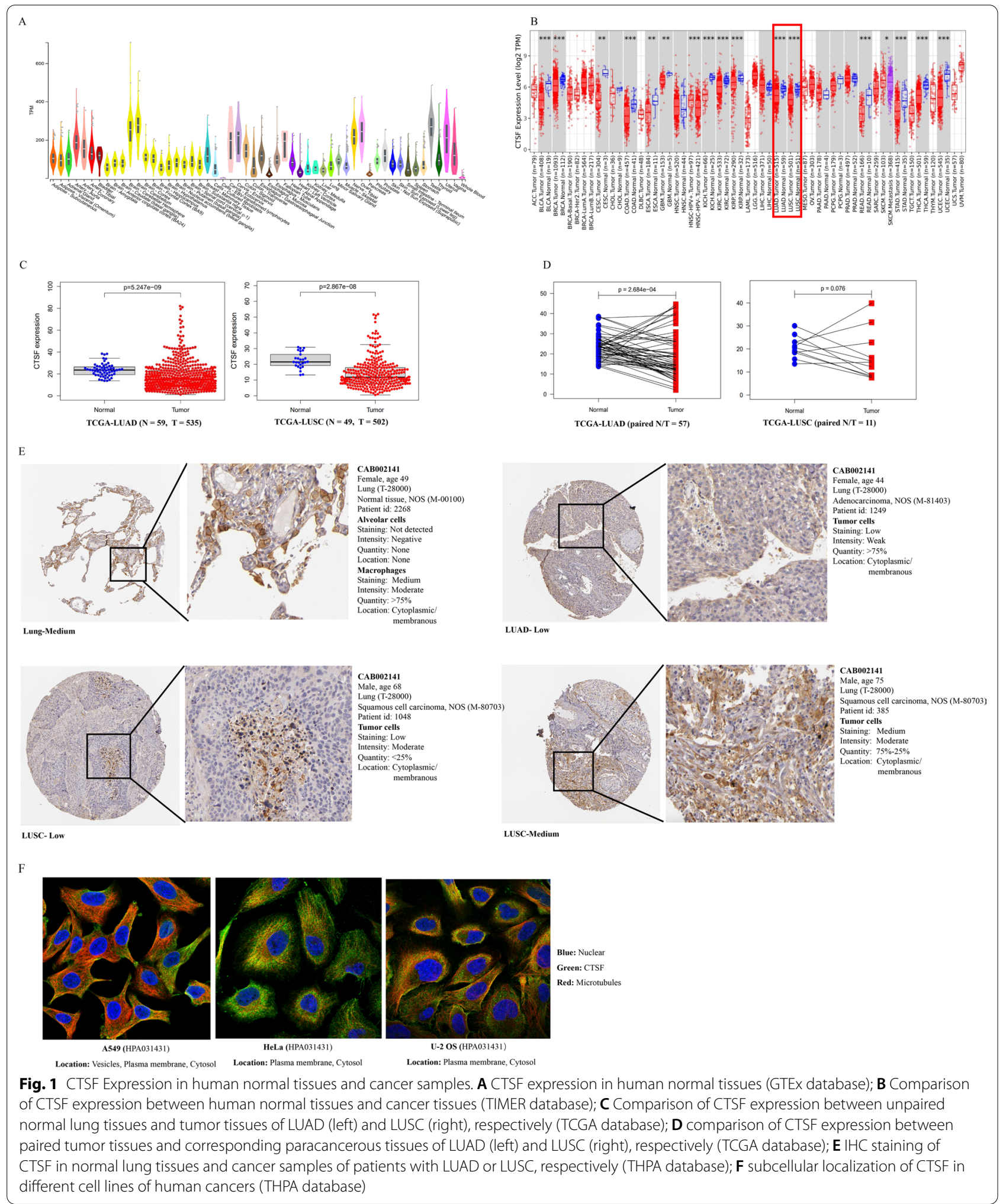


Table 1 Correlation between CTSF expression and that of immune cell markers using TIMER

\begin{tabular}{|c|c|c|c|c|c|c|c|c|c|}
\hline \multirow[t]{3}{*}{ Description } & \multirow[t]{3}{*}{ Gene markers } & \multicolumn{4}{|c|}{$\operatorname{LUAD}(n=515)$} & \multicolumn{4}{|c|}{ LUSC $(n=501)$} \\
\hline & & \multicolumn{2}{|l|}{ None } & \multicolumn{2}{|l|}{ Purity } & \multicolumn{2}{|l|}{ None } & \multicolumn{2}{|l|}{ Purity } \\
\hline & & cor & $p$ & cor & $p$ & cor & $p$ & cor & $p$ \\
\hline \multirow[t]{2}{*}{$\mathrm{CD}^{+}{ }^{+} \mathrm{T}$ cell } & CD8A & -0.075 & 0.088 & -0.092 & * & 0.104 & $*$ & 0.101 & $*$ \\
\hline & CD8B & -0.033 & 0.948 & -0.015 & 0.739 & 0.229 & $* * *$ & 0.220 & $* * *$ \\
\hline \multirow[t]{3}{*}{ T cell (general) } & CD3D & -0.016 & 0.709 & -0.020 & 0.651 & 0.076 & 0.090 & 0.068 & 0.139 \\
\hline & CD3E & 0.032 & 0.473 & 0.042 & 0.353 & 0.118 & $* *$ & 0.118 & $* *$ \\
\hline & CD2 & 0.020 & 0.648 & 0.025 & 0.577 & 0.107 & $*$ & 0.102 & * \\
\hline \multirow[t]{2}{*}{ B cell } & CD19 & 0.139 & $* *$ & 0.176 & $* * *$ & 0.115 & $*$ & 0.115 & * \\
\hline & CD79A & 0.157 & $* * *$ & 0.191 & $* * *$ & 0.154 & $* * *$ & 0.168 & $* * *$ \\
\hline \multirow[t]{2}{*}{ Monocyte } & CD86 & 0.039 & 0.372 & 0.004 & 0.388 & 0.130 & $*$ & 0.125 & 0.006 \\
\hline & CD115(CSF1R) & 0.133 & $* *$ & 0.145 & $* *$ & 0.186 & $* * *$ & 0.197 & $* * *$ \\
\hline \multirow[t]{3}{*}{ TAM } & $\mathrm{CCL} 2$ & 0.085 & 0.053 & 0.094 & $*$ & 0.215 & $* * *$ & 0.211 & $* * *$ \\
\hline & CD68 & 0.008 & 0.860 & 0.013 & 0.776 & 0.052 & 0.245 & 0.038 & 0.413 \\
\hline & IL10 & 0.093 & * & 0.112 & * & 0.186 & $* * *$ & 0.190 & $* * *$ \\
\hline \multirow[t]{3}{*}{ M1 Macrophage } & INOS(NOS2) & 0.096 & * & 0.109 & * & 0.135 & $* *$ & 0.121 & $* *$ \\
\hline & IRF5 & 0.025 & 0.564 & 0.031 & 0.492 & 0.015 & 0.740 & 0.009 & 0.840 \\
\hline & COX2(PTGS2) & 0.046 & 0.295 & 0.037 & 0.407 & 0.103 & * & 0.095 & * \\
\hline \multirow[t]{3}{*}{ M2 Macrophage } & CD163 & 0.012 & 0.794 & 0.009 & 0.843 & 0.135 & $* *$ & 0.130 & $* *$ \\
\hline & VSIG4 & 0.024 & 0.592 & 0.032 & 0.480 & 0.140 & $* *$ & 0.134 & $* *$ \\
\hline & MS4A4A & 0.041 & 0.355 & 0.050 & 0.263 & 0.118 & $* *$ & 0.110 & * \\
\hline \multirow[t]{3}{*}{ Neutrophils } & CD66B(CEACAM8) & 0.187 & $* * *$ & 0.202 & $* * *$ & 0.130 & $* *$ & 0.109 & * \\
\hline & CD11B(ITGAM) & 0.100 & $*$ & 0.109 & $*$ & 0.163 & $* * *$ & 0.164 & $* * *$ \\
\hline & CCR7 & 0.118 & $* *$ & 0.152 & $* * *$ & 0.153 & $* * *$ & 0.153 & $* * *$ \\
\hline \multirow[t]{7}{*}{ Natural killer cell } & KIR2DL1 & -0.083 & 0.060 & -0.095 & $*$ & 0.030 & 0.051 & 0.021 & 0.652 \\
\hline & KIR2DL3 & -0.145 & $* * *$ & -0.143 & $* *$ & 0.019 & 0.677 & 0.012 & 0.796 \\
\hline & KIR2DL4 & -0.213 & $* * *$ & -0.236 & $* * *$ & 0.001 & 0.986 & -0.010 & 0.890 \\
\hline & KIR3DL1 & -0.081 & 0.066 & -0.086 & 0.556 & 0.103 & * & 0.087 & 0.058 \\
\hline & KIR3DL2 & -0.108 & * & -0.121 & $* *$ & -0.021 & 0.634 & -0.034 & 0.465 \\
\hline & KIR3DL3 & -0.151 & $* * *$ & -0.154 & $* * *$ & 0.040 & 0.377 & 0.036 & 0.434 \\
\hline & KIR2DS4 & -0.071 & 0.108 & -0.073 & 0.105 & 0.027 & 0.550 & 0.024 & 0.600 \\
\hline \multirow[t]{7}{*}{ Dendritic cell } & HLA-DPB1 & 0.186 & $* * *$ & 0.208 & $* * *$ & 0.156 & $* * *$ & 0.168 & $* * *$ \\
\hline & HLA-DQB1 & 0.134 & $* *$ & 0.155 & $* * *$ & 0.095 & $*$ & 0.098 & $*$ \\
\hline & HLA-DRA & 0.156 & $* * *$ & 0.173 & $* * *$ & 0.140 & $* *$ & 0.145 & $* *$ \\
\hline & HLA-DPA1 & 0.179 & $* * *$ & 0.197 & $* * *$ & 0.151 & $* * *$ & 0.157 & $* * *$ \\
\hline & BDCA-1(CD1C) & 0.197 & $* * *$ & 0.217 & $* * *$ & 0.101 & * & 0.103 & * \\
\hline & BDCA-4(NRP1) & 0.061 & 0.168 & 0.059 & 0.190 & 0.262 & $* * *$ & 0.184 & $* * *$ \\
\hline & CD11C(ITGAX) & 0.056 & 0.203 & 0.059 & 0.191 & 0.088 & * & 0.094 & $*$ \\
\hline \multirow[t]{5}{*}{ Th1 } & T-bet(TBX21) & -0.029 & 0.514 & -0.026 & 0.566 & 0.078 & 0.082 & 0.078 & 0.090 \\
\hline & STAT4 & 0.051 & 0.248 & 0.056 & 0.213 & 0.189 & $* * *$ & 0.211 & $* * *$ \\
\hline & STAT1 & -0.121 & $* *$ & -0.144 & $* *$ & 0.031 & 0.495 & 0.026 & 0.569 \\
\hline & IFN- $\gamma($ IFNG) & -0.206 & $* * *$ & -0.225 & $* * *$ & -0.051 & 0.256 & -0.034 & 0.462 \\
\hline & TNF-a (TNF) & 0.048 & 0.274 & 0.061 & 0.173 & 0.075 & 0.092 & 0.067 & 0.142 \\
\hline \multirow[t]{4}{*}{ Th2 } & GATA3 & 0.071 & 0.106 & 0.075 & 0.097 & 0.310 & $* * *$ & 0.307 & $* * *$ \\
\hline & STAT6 & 0.102 & $* *$ & 0.113 & * & 0.043 & 0.340 & 0.042 & 0.358 \\
\hline & STAT5A & 0.167 & $* * *$ & 0.183 & $* * *$ & 0.245 & $* * *$ & 0.259 & $* * *$ \\
\hline & IL13 & 0.024 & 0.588 & 0.024 & 0.598 & 0.033 & 0.466 & 0.020 & 0.656 \\
\hline Tfh & BCL6 & 0.188 & $* * *$ & 0.192 & $* * *$ & -0.031 & 0.486 & -0.034 & 0.458 \\
\hline & IL21 & -0.071 & 0.108 & -0.071 & 0.115 & -0.044 & 0.323 & -0.051 & 0.264 \\
\hline
\end{tabular}


Table 1 (continued)

\begin{tabular}{|c|c|c|c|c|c|c|c|c|c|}
\hline \multirow[t]{3}{*}{ Description } & \multirow[t]{3}{*}{ Gene markers } & \multicolumn{4}{|c|}{ LUAD $(n=515)$} & \multicolumn{4}{|c|}{ LUSC $(n=501)$} \\
\hline & & \multicolumn{2}{|l|}{ None } & \multicolumn{2}{|l|}{ Purity } & \multicolumn{2}{|l|}{ None } & \multicolumn{2}{|l|}{ Purity } \\
\hline & & cor & $p$ & cor & $p$ & cor & $p$ & cor & $p$ \\
\hline \multirow[t]{2}{*}{ Th17 } & STAT3 & 0.206 & $* * *$ & 0.213 & $* * *$ & 0.189 & $* * *$ & 0.187 & $* * *$ \\
\hline & IL17A & -0.132 & $* *$ & -0.126 & $* *$ & -0.128 & $* *$ & -0.119 & $* *$ \\
\hline \multirow[t]{4}{*}{ Treg } & FOXP3 & 0.050 & 0.262 & 0.059 & 0.189 & 0.168 & $* * *$ & 0.176 & $* * *$ \\
\hline & CCR8 & 0.023 & 0.596 & 0.018 & 0.696 & 0.151 & $* * *$ & 0.142 & $* *$ \\
\hline & STAT5B & 0.286 & $* * *$ & 0.293 & $* * *$ & 0.287 & $* * *$ & 0.289 & $* * *$ \\
\hline & TGF $\beta$ (TGFB1) & 0.171 & $* * *$ & 0.181 & $* * *$ & 0.084 & 0.059 & 0.075 & 0.102 \\
\hline \multirow[t]{5}{*}{ T cell exhaustion } & PD-1 (PDCD1) & 0.002 & 0.958 & 0.001 & 0.991 & 0.137 & $* *$ & 0.146 & $* *$ \\
\hline & CTLA4 & -0.039 & 0.378 & -0.048 & 0.289 & 0.079 & 0.078 & 0.074 & 0.107 \\
\hline & LAG3 & -0.080 & 0.069 & -0.098 & $*$ & 0.057 & 0.201 & 0.051 & 0.264 \\
\hline & TIM-3 (HAVCR2) & 0.002 & 0.969 & 0.001 & 0.984 & 0.093 & * & 0.085 & 0.065 \\
\hline & GZMB & -0.239 & $* * *$ & -0.273 & $* * *$ & 0.009 & 0.848 & -0.006 & 0.901 \\
\hline
\end{tabular}

Function, Fig. 3C). KEGG analysis demonstrated genes co-expressed positively with CTSF in LUAD were primarily enriched in Valine, leucine and isoleucine degradation, Asthma and Cell adhesion molecules (CAMs), while genes co-expressed negatively with CTSF in LUAD were primarily enriched in Cell cycle, ribosome biogenesis in eukaryotes, spliceosome, RNA transport, proteasome and others (Fig. 3D). GO term annotation displayed that molecular function of genes co-expressed positively with CTSF in LUSC were mainly involved in extracellular matrix structure constituent, collagen binding, actinin binding, fibronectin binding, transmembrane receptor protein kinase activity, structure constituent of muscle, hydrolase activity, acting on glycosyl bonds, glycosaminoglycan binding, coreceptor activity and Wnt-protein binding. In contrast, molecular function of genes coexpressed negatively with CTSF in LUSC were mainly involved in single-stranded DNA binding, RNA polymerase binding, DNA secondary structure binding and others (Fig. 3E). KEGG analysis showed genes co-expressed positively with CTSF in LUSC were primarily enriched in ECM-receptor interaction, Morphine addiction, Malaria, Other glycan degradation, Renin secretion, Glycosphingolipid biosynthesis, Vascular smooth muscle contraction, and Dilated cardiomyopathy (DCM), while genes co-expressed negatively with CTSF in LUSC were primarily enriched in Ribosome biogenesis in eukaryotes, Spliceosome, Proteasome and others (Fig. 3F).

Gene-gene interaction network for CTSF constructed using GeneMANIA showed that CTSF had similar function as other 20 genes (MAP1LC3A, CD74, ITPKB, CTSW, ABHD14A, CTSH, CTSV, CTSS, CTSK, MAPK14, CTSL, CTSO, SHOC2, GAL3ST1, OTUD7B, ZNF334, TINAGL1, CTSC, CTSB, and CDIPT) (Fig. 3G).
Functional analysis indicated that proteins encoded by these 20 genes were significantly correlated with following terms: cysteine-type peptidase activity, cysteinetype endopeptidase activity, lysosomal lumen, vacuolar lumen, antigen processing and presentation of exogenous peptide antigen via MHC class II, antigen processing and presentation, and adaptive immune response. PPI network constructed using STRING showed that 10 proteins were interacted with CTSF (Fig. 3H). Among these proteins interacted with CTSF, HLA-DQA1, HLA-DPA1, HLA-DRA, HLA-DQA2, HLA-DRB1, HLA-DQB1, HLA-DRB5, HLA-DQB2 and HLA-DPB1 are different subunits of the human leukocyte antigen (HLA) class II, which plays a central role in immune system by presenting peptides derived from extracellular proteins. CD74 is associates with $\mathrm{MHC}$ and also regulates antigen presentation for immune response.

\section{CTSF might regulate immune infiltration of NSCLC}

In order to explore the role of CTSF in immune response of NSCLC, TCGA-LUAD and TCGA-LUSC cohorts were downloaded to explore association of CTSF expression with infiltrating levels of immune cells. Landscape of infiltrating immune cells in cancerous and healthy biopsies for TCGA-LUAD and TCGA-LUSC cohorts was shown in Fig. 4A (above: LUAD, below: LUSC). As shown in Fig. 4A and $4 \mathrm{~B}$, proportions of $\mathrm{B}$ cells, Natural killer (NK) cells and uncharacterized cells were significantly higher while that of Macrophages, $\mathrm{CD} 4^{+} \mathrm{T}$ cells and Endothelial cells were significantly lower in tissues of LUAD related to normal lung tissues. In LUSC, proportions of $\mathrm{CD}^{+} \mathrm{T}$ cells, $\mathrm{B}$ cells, NK cells and uncharacterized cells were significantly higher while that of Macrophages and Endothelial cell were 


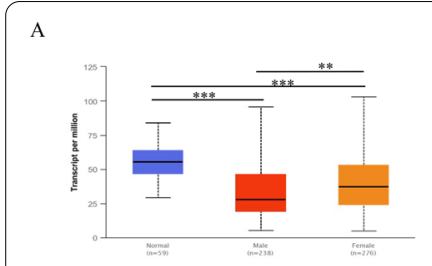

CTSF expression in LUAD based on patient's gender

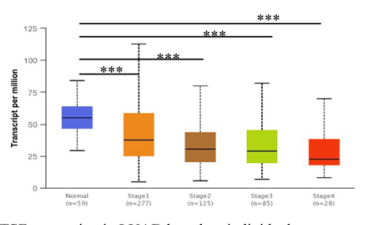

CTSF expression in LUAD based on individual cancer stages

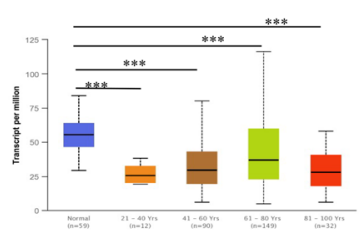

CTSF expression in LUAD based on patient's age

CTSF expression in LUAD based on Histological subtypes
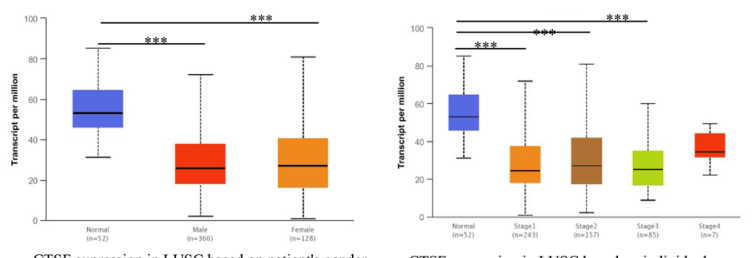

CTSF expression in LUSC based on individual cancer

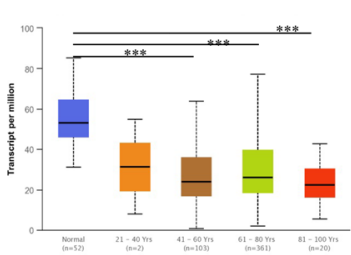

CTSF expression in LUSC based on Histological subtypes

CTSF expression in LUSC based on patient's age

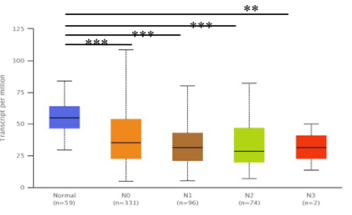

CTSF expression in LUAD based on nodal metastasis status

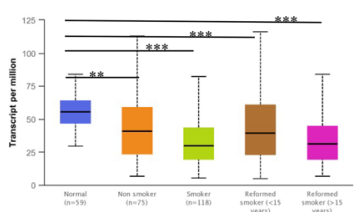

CTSF expression in LUAD based on patient's smoking habits
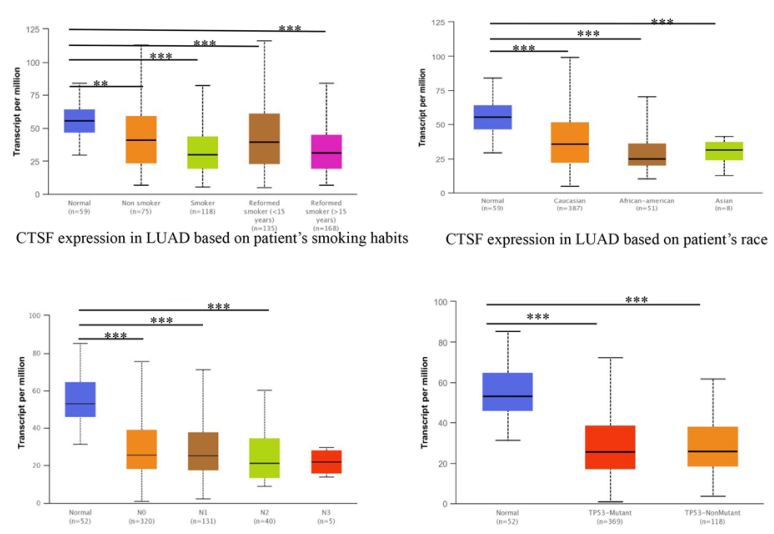

CTSF expression in LUAD based on patient's race

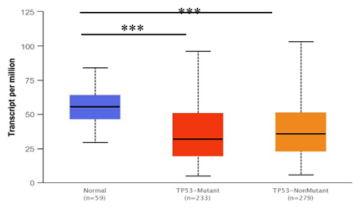

CTSF expression in LUAD based on TP53 mutation status

CTSF expression in LUSC based on nodal metastasis status CTSF expression in LUSC based on TP53 mutation status
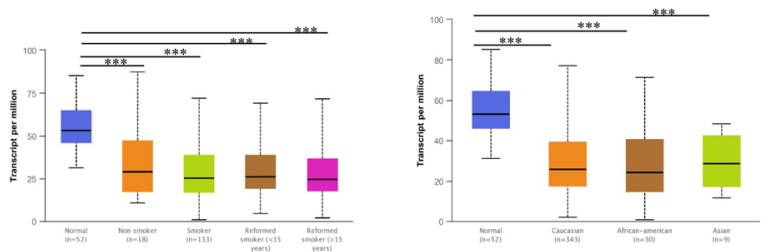

CTSF expression in LUSC based on patient's race

CTSF expression in LUSC based on patient's smoking habits

C

D

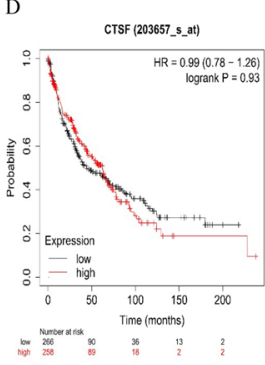

os

F

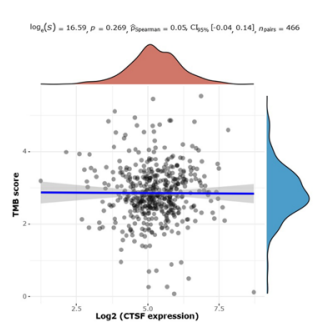

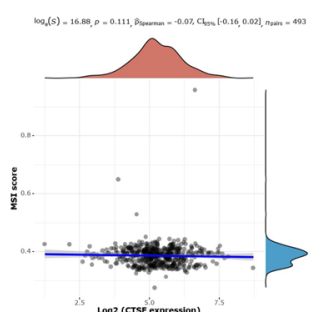

Log2 (crstio expression) is

Fig. 2 Associations of CTSF expression with clinical characteristics of NSCLC. A, B Associations of CTSF expression with clinical characteristics of LUAD and LUSC, respectively; C, D associations of CTSF expression with prognosis of LUAD (C) and LUSC (D), respectively; $\mathbf{E}$, $\mathbf{F}$ Correlations of CTSF expression with TMB and MSI in LUAD $(\mathbf{E})$ and LUSC $(\mathbf{F})$, respectively 
significantly lower in tumor tissues compared with that of normal tissues.

TIMER analysis demonstrated that infiltration levels of B cells, $\mathrm{CD}^{+} \mathrm{T}$ cells, Macrophages, Neutrophils, and Dendritic cells (DCs) increased with copy numbers of CTSF gene both in LUAD and LUSC (Fig. 4C). Additionally, after adjusted by tumor purity, CTSF expression was shown to be positively correlated with immune infiltration of $\mathrm{B}$ cells $(\mathrm{r}=0.212, p=2.59 \mathrm{e}-06), \mathrm{CD} 4^{+} \mathrm{T}$ cells $(\mathrm{r}=0.179$, $p=7.83 \mathrm{e}-05)$, Macrophage $(\mathrm{r}=0.133, \quad p=3.38 \mathrm{e}-03)$, and DCs $(\mathrm{r}=0.124, p=5.93 \mathrm{e}-03)$ in LUAD (Fig. 4D). In LUSC, besides B cells $(\mathrm{r}=0.204, p=8.17 \mathrm{e}-06), \mathrm{CD} 4^{+} \mathrm{T}$ cells $(\mathrm{r}=0.257, p=1.35 \mathrm{e}-08)$, Macrophage $(\mathrm{r}=0.282$, $p=3.49 \mathrm{e}-10)$, and DCs $(\mathrm{r}=0.189, p=3.41 \mathrm{e}-05)$, immune infiltration of $\mathrm{CD}^{+} \mathrm{T}$ cells $(\mathrm{r}=0.132, p=3.82 \mathrm{e}-03)$ and Neutrophil $(\mathrm{r}=0.104, p=2.34 \mathrm{e}-02)$ were also found to be positively correlated with CTSF expression (Fig. 4E).

Additionally, the expression analysis between CTSF and molecular marker of immunological cells showed that CTSF expression was positively related with that of molecular markers of B cells, Monocytes, TAMs, M1 Macrophages, Neutrophils, DCs, Th2 cells, Tfh cells, Th17 cells and Treg cells, while negatively related with that of $\mathrm{CD} 8^{+} \mathrm{T}$ cells, NK cells, Th1 cells and Th17 cells in LUAD. In LUSC, CTSF expression was shown to be positively associated with that of $\mathrm{CD}^{+} \mathrm{T}$ cells, general $\mathrm{T}$ cells, $\mathrm{B}$ cells, Monocytes, TAMs, M1 macrophages, M2 macrophages, Neutrophils, DCs, Th1 cells, Th2 cells, Th17 cells and Treg cells, while negatively related with that of Th17 cells (Table 1).

\section{CTSF-related immunomodulators were associated with prognosis of patients with NSCLC}

To further confirm association of CTSF with immune response of NSCLC, CTSF expression was analyzed with that of the known immunomodulators using TISIDB, which revealed that CTSF expression was significantly associated with that of 40 immunomodulators (including 28 immunostimulators and 12 immunoinhibitors) in LUAD or LUSC (Fig. 4F, Tables 2 and 3). Among the 12 immunoinhibitors, CTSF expression was negatively related with that of CD244 (rho $=-0.115, p=8.92 \mathrm{e}-03)$, CD274 (also PD-L1, rho $=-0.177, p=5.53 \mathrm{e}-05$ ), CTLA4 (rho $=-0.140, p=1.46 \mathrm{e}-03)$, HAVCR2 $(\mathrm{rho}=-0.087$, $p=0.047)$, LAG3 (rho $=-0.163, p=2.03 \mathrm{e}-04)$, PDCD1 (also PD-1, rho $=-0.089, p=0.044$ ), PVRL2 (rho $=-0.102$, $p=0.020$ ), and TIGIT (rho $=-0.151, p=5.94 \mathrm{e}-04$ ), while positively related with that of KDR $(\mathrm{rho}=0.131$, $p=2.97 \mathrm{e}-03)$ and VTCN1 (rho $=0.157, p=3.43 \mathrm{e}-04)$ in
LUAD. Of the 28 immunostimulators, CTSF expression was negatively related with that of CD276 (rho $=-0.088$, $p=0.045)$, ICOS (rho $=-0.120, p=6.55 \mathrm{e}-03)$, IL2RA (rho $=-0.137, \quad p=1.78 \mathrm{e}-03), \quad$ IL6 $\quad($ rho $=-0.160$, $p=2.63 \mathrm{e}-04), \quad$ KLRC1 $\quad(\mathrm{rho}=-0.181, \quad p=3.57 \mathrm{e}-05)$, KLRK1 (rho $=-0.113, p=0.010)$, MICB (rho $=-0.165$, $p=1.63 \mathrm{e}-04)$, PVR (rho $=-0.263, p=1.55 \mathrm{e}-09)$, RAETIE (rho $=-0.175, p=6.40 \mathrm{e}-05)$, TMIGD2 (rho $=-0.104$, $p=0.018), \quad$ TNFRSF9 $\quad($ rho $=-0.141, \quad p=1.29 \mathrm{e}-03)$, TNFSF4 $(\mathrm{rho}=-0.090, \quad p=0.042)$ and TNFSF9 $(\mathrm{rho}=-0.143, \quad p=1.13 \mathrm{e}-03)$, while positively related with that of CD27 (rho=0.102, $p=0.021$ ), CD40LG (rho $=0.132, \quad p=2.63 \mathrm{e}-03), \quad$ CXCL12 $\quad($ rho $=0.167$, $p=1.42 \mathrm{e}-04)$, ENTPD1 (rho $=0.101, p=0.022)$, ICOSLG $($ rho $=0.106, p=0.016)$, IL6R $($ rho $=0.124, p=4.77 \mathrm{e}-03)$, TMEM173 $\quad($ rho $=0.158, \quad p=3.19 \mathrm{e}-04), \quad$ TNFRSF13B (rho $=0.129, p=3.24 \mathrm{e}-03$ ), TNFRSF17 (also known as BCMA, rho $=0.126, p=0.004)$ and TNFSF13 $(\mathrm{rho}=0.089$, $p=0.043$ ). In LUSC, 18 immunomodulators (including 14 immunostimulators and four immunoinhibitors) were significantly associated with CTSF expression. Of the four immunoinhibitors, CTSF expression was negatively related with that of $\mathrm{CD} 274$ (rho $=-0.208, p=2.75 \mathrm{e}-06$ ), while positively associated with that of ADORA2A ( $\mathrm{rho}=0.174$, $p=9.29 \mathrm{e}-05), \quad$ IL10 $\quad($ rho $=0.133, \quad p=2.95 \mathrm{e}-03) \quad$ and KDR (rho $=0.091, p=0.043$ ). Of the 14 immunostimulators, CTSF expression was positively with that of CD27 (rho $=0.103, p=0.022), \mathrm{CD} 28$ (rho $=0.100, p=0.025)$, $\mathrm{CD} 40$ (rho $=0.193, p=1.41 \mathrm{e}-05), \mathrm{CD} 40 \mathrm{LG}(\mathrm{rho}=0.100$, $p=0.026), \quad$ CD70 (rho $=0.135, \quad p=2.5 \mathrm{e}-03), \quad$ CXCL12 (rho $=0.150, p=7.81 \mathrm{e}-04), \quad$ ENTPD1 $\quad($ rho $=0.175$, $p=8.49 \mathrm{e}-05), \quad$ TNFRSF14 $(\mathrm{rho}=0.159, p=3.71 \mathrm{e}-04)$, TNFRSF17 (rho $=0.103, p=0.021)$, TNFRSF8 $(\mathrm{rho}=0.090$, $p=0.0446)$ and TNFSF4 (rho $=0.099, p=0.0268$ ), while negatively related with that of RAET1E (rho $=-0.089$, $p=0.046)$, TNFSF18 (rho $=-0.154, p=5.5 \mathrm{e}-04)$ and TNFSF9 (rho $=-0.102, p=0.0237$ ) (Tables 2 and 3 ).

Subsequent analysis of immunomodulators with prognosis of NSCLC patients using Kaplan-Meier plotter displayed that high expression of four immunostimulators (TMEM173, $\mathrm{HR}=0.58, p=9.6 \mathrm{e}-06$; IL6R, $\mathrm{HR}=0.36, p=5.0 \mathrm{e}-15$; ICOS, $\mathrm{HR}=0.49, p=5.4 \mathrm{e}-09$; and ENTPD1, HR $=0.62, p=1.6 \mathrm{e}-04)$ and one immunoinhibitor (CTLA4, HR=0.57, $\mathrm{p}=5.6 \mathrm{e}-06$ ) were related to good prognosis of LUAD patients (Fig. 4G). In contrast, high expression of seven immunostimulators (TNFSF13, $\mathrm{HR}=1.89, p=1.3 \mathrm{e}-07 ; \mathrm{CD} 276, \mathrm{HR}=1.74$, $p=7.2 \mathrm{e}-06 ; \quad$ IL6, $\quad \mathrm{HR}=1.69, \quad p=9.9 \mathrm{e}-06 ; \quad \mathrm{CD} 27$,

(See figure on next page.)

Fig. 3 Genes Co-expressed with CTSF in NSCLC (LinkedOmics). A, B Heatmaps showing the top 50 genes positively (left) or negatively (right) co-expressed genes with CTSF in LUAD (A) and LUSC (B), respectively; C, D enriched genes co-expressed with CTSF in LUAD using GO (C) and KEGG (D) analysis, respectively; E, F enriched genes co-expressed with CTSF in LUSC using GO (E) and KEGG (F) analysis, respectively; G Genegene interaction network constructed using GeneMANIA; $(\mathrm{H})$ PPI network constructed by STRING (medium confidence $=0.400$ ) 

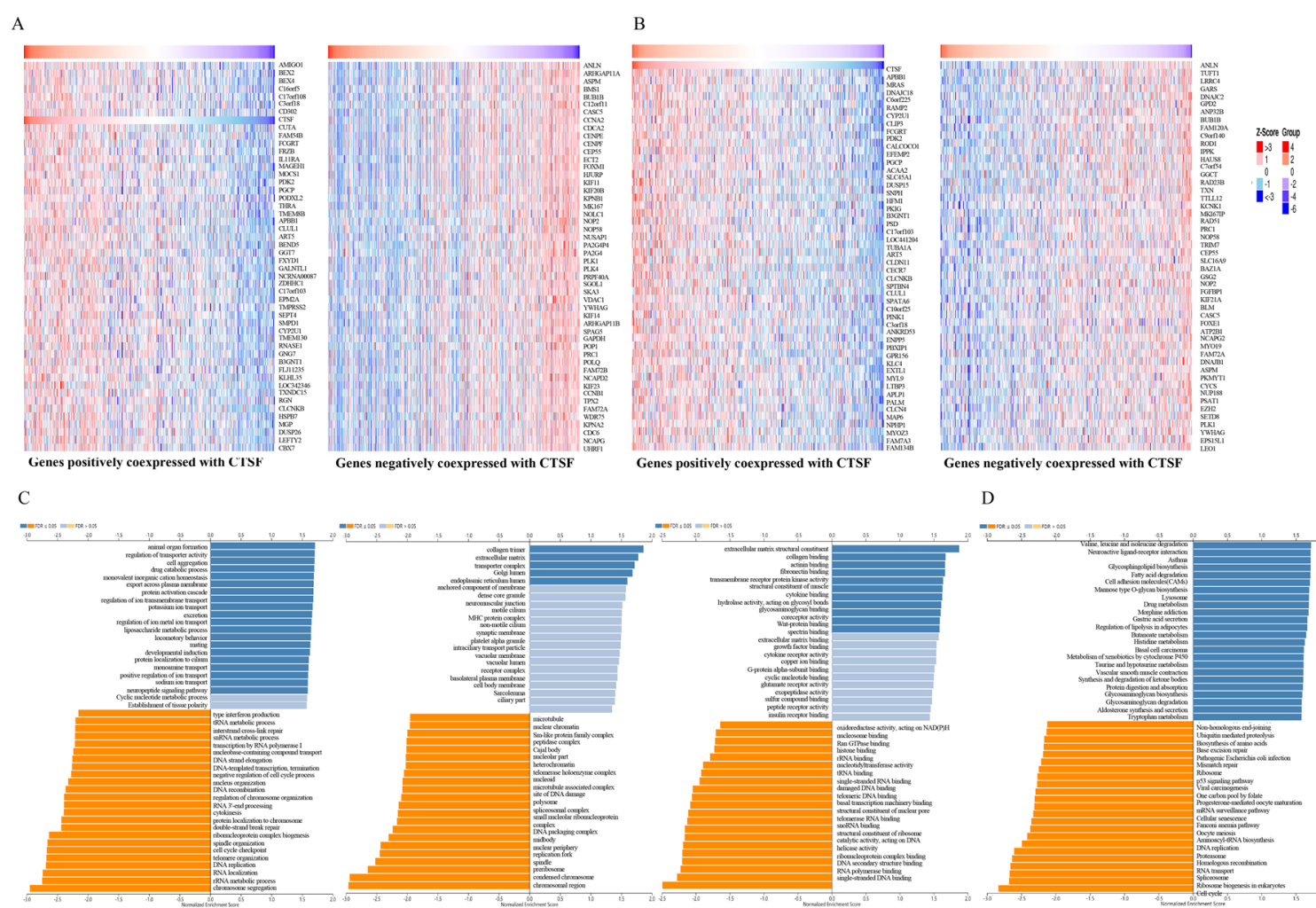

D

GO-Biological process

GO-Cellular Component

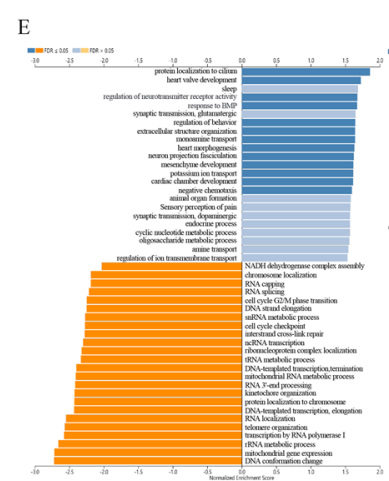

GO-Biological process

G

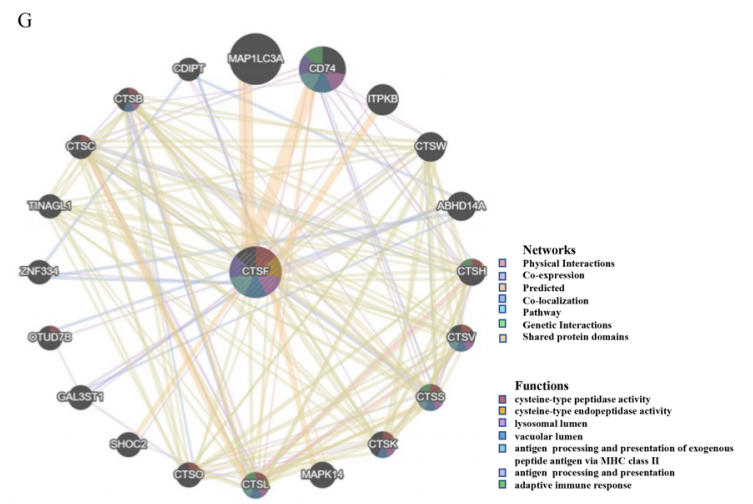

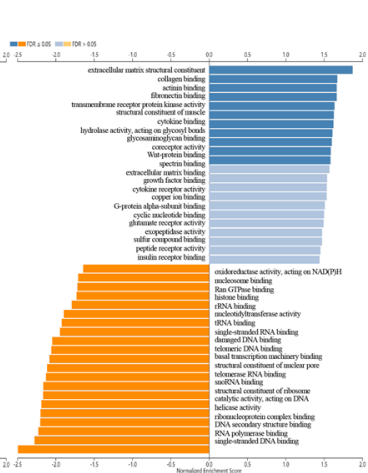

GO-Molecular Function
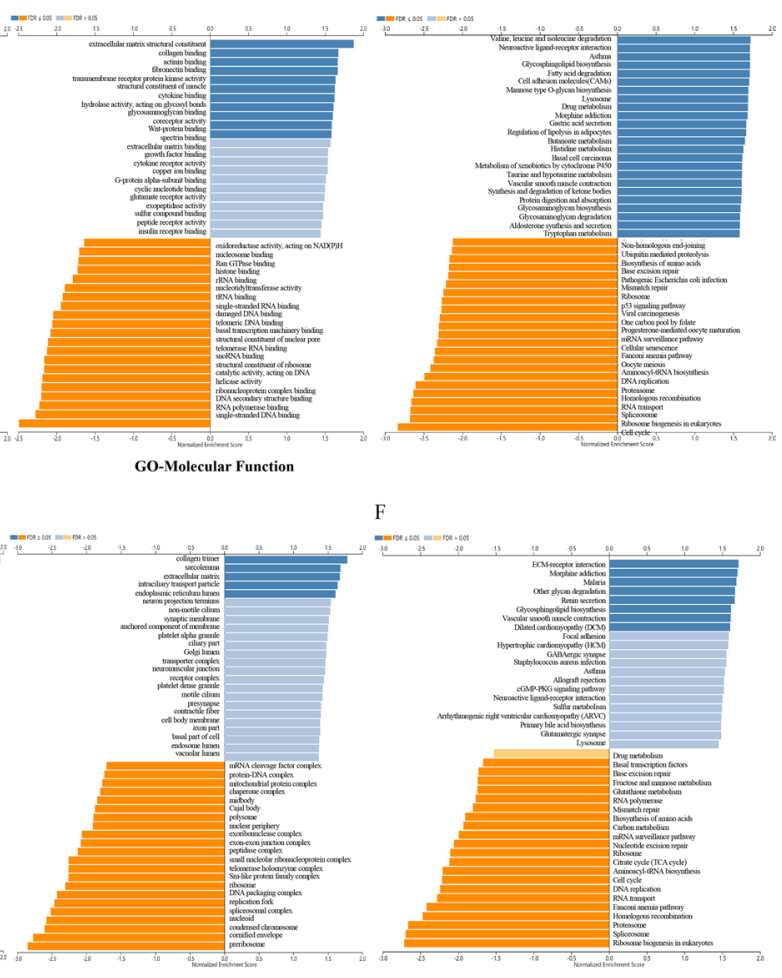

GO-Molecular Function

H

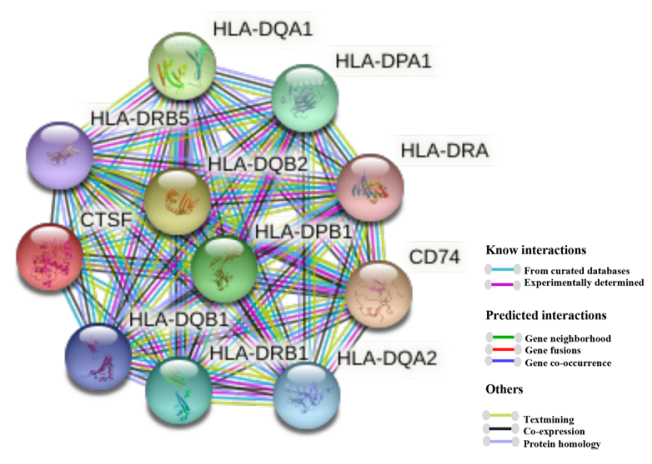

Fig. 3 (See legend on previous page.) 
$\mathrm{HR}=1.38, p=6.7 \mathrm{e}-03 ; \mathrm{PVR}, \mathrm{HR}=1.54, p=5.0 \mathrm{e}-04$; TNFSF9, $\mathrm{HR}=1.5, p=6.5 \mathrm{e}-04 ; \mathrm{TMIGD} 2, \mathrm{HR}=1.71$, $p=1.3 \mathrm{e}-05)$ and one immunoinhibitor (LAG3, $\mathrm{HR}=1.34, p=1.3 \mathrm{e}-02$ ) were associated with poor prognosis of LUAD patients (Fig. $4 \mathrm{H}$ ). Of these immunomodulators, a high expression of two immunostimulators (CD27, $\mathrm{HR}=0.75, p=0.018$, and TNFRSF17 (also $\mathrm{BCMA}), \mathrm{HR}=0.68, p=0.0015)$ were found to be related with a good prognosis of LUSC patients (Fig. 4I).

\section{Discussion}

CTSF is unique in human cathepsins because of having an extended $\mathrm{N}$-terminal anterior region containing a cystatin domain. Prior studies demonstrated that CTSF may contribute to the progression of gastric cancer, pediatric brain tumors, breast cancer, and lymphoma/leukemia as a suppressor gene [25-28]. Presently, the role of CTSF in NSCLC is still unclear. In the study, the Bioinformatics assessment showed that CTSF might also function as a tumor suppressor gene in NSCLC via regulating immune responses.

Exploration of CTSF expression in TIMER and TCGA datasets showed that CTSF was downregulated in lung cancer tissues related to that of normal lung tissues. Analysis of CTSF expression with clinical characteristics of LUAD patients demonstrated that CTSF expression decreased with stage, metastasis and smoking. High expressed CTSF was significantly correlated with better OS and FS in LUAD patients and better FS in LUSC patients, indicating that CTSF might be a tumor suppressor gene both in LUAD and LUSC, similar to its function in gastric cancer, brain tumors and lymphoma/ leukemia. In gastric cancer, downregulated CTSF was found to induce proliferation and inhibit apoptosis of gastric cancer cells [25]. Conversely, progression of gastric cancer was inhibited by upregulated CTSF promoted by LINC00982 binding to transcription factor HEY1 [26]. In brain tumors, CTSF was shown to be lower in ependymoma, glioblastoma, and medulloblastoma compared to normal brain [27]. CTSF knockdown was reported to promote lymphoma/leukemia development when PUMA and p21 were absent [28]. Moreover, CTSF was shown to be one of genes encoding components of the degradome which were reprogramed in acquired resistance to metformin in breast cancer cells [29].

Exploration of CTSF as a tumor suppressor gene in NSCLC by constructing gene-gene interaction network and PPI network demonstrated that besides potential functions of cysteine-type peptidase activity, cysteinetype endopeptidase activity, lysosomal lumen, and vacuolar lumen, CTSF also functions in antigen processing and presentation of exogenous peptide antigen via $\mathrm{MHC}$ class II, antigen processing and presentation, and adaptive immune response. The findings were consistent with that of IHC staining of CTSF in normal lung tissues and tumor tissues of NSCLC, which showed that medium staining of CTSF was observed in macrophage cells rather than alveolar cells in normal lung tissues or tumor tissues. Notably, CTSF straining was shown to be stronger in macrophages and other infiltrating immune cells than that of tumor cells in cancerous tissues of NSCLC. Additionally, among the ten proteins interacted with CTSF, nine proteins (HLA-DQA1, HLA-DPA1, HLA-DRA, HLA-DQA2, HLA-DRB1, HLA-DQB1, HLA-DRB5, HLA-DQB2 and HLA-DPB1) are the different subunits of HLA class II, which plays a central role in immune system by presenting peptides derived from extracellular proteins [30]. The other one CTSF-interacted protein CD74 is associates with $\mathrm{MHC}$ and also regulates antigen presentation for immune response [31]. The findings mentioned above strongly suggested that CTSF might function as a tumor suppressor gene via contributing to antigen presentation for immune response of NSCLC. Subsequent analysis showing CTSF expression was positively related with immune infiltration of B cells, macrophage, DCs, CD8 ${ }^{+} \mathrm{T}$ cells, $\mathrm{CD} 4^{+} \mathrm{T}$ cells and Neutrophil in LUAD or LUSC provided evidences that CTSF contributed to immune response in NSCLC.

Contribution of CTSF to immune responses of NSCLC was confirmed by expression correlation of CTSF with immune marker sets and immunomodulators in NSCLC. Expression analysis of CTSF with immune marker sets showed that CTSF expression was positively related with that of molecular markers of B cells, Monocytes, TAMs, M1 macrophages, Neutrophils, DCs, Th2 cells, Tfh cells, Th17 cells and Treg cells in LUAD. In LUSC, CTSF expression was positively associated with that of

(See figure on next page.)

Fig. 4 Correlation of CTSF expression with infiltration levels of immune cells and immunomodulators in NSCLC. A Landscape of infiltrating immune cells in cancerous and healthy biopsies for TCGA-LUAD and TCGA-LUSC cohorts, respectively; B EPIC scores of immune infiltrating cells between normal lung tissues and LUAD or LUSC tissues, respectively; $\mathbf{C}$ association of CTSF copy numbers with immune cell infiltration levels in LUAD (above) and LUSC (below) cohorts, respectively; D, E Correlation between CTSF expression and immune cells in LUAD (D) and LUSC (E), respectively; F Heatmap of CTSF-related immunomodulators in LUAD and LUSC, respectively; G association of CTSF-related immunostimulators (TMEM173, IL6R, ICOS, and ENTPD1) and CTSF-related immunoinhibitor (CTLA4) with favorable prognosis of LUAD patients; H Association of CTSF-related immunostimulators (TNFSF13, CD276, IL6, CD27, PVR, TNFSF9, TMIGD2) and CTSF-related immunoinhibitor (LAG3) with poor prognosis of LUAD patients; (I) Relationship of CTSF-related immunostimulators CD27 and TNFRSF17 (also BCMA) with good prognosis of LUSC patients 


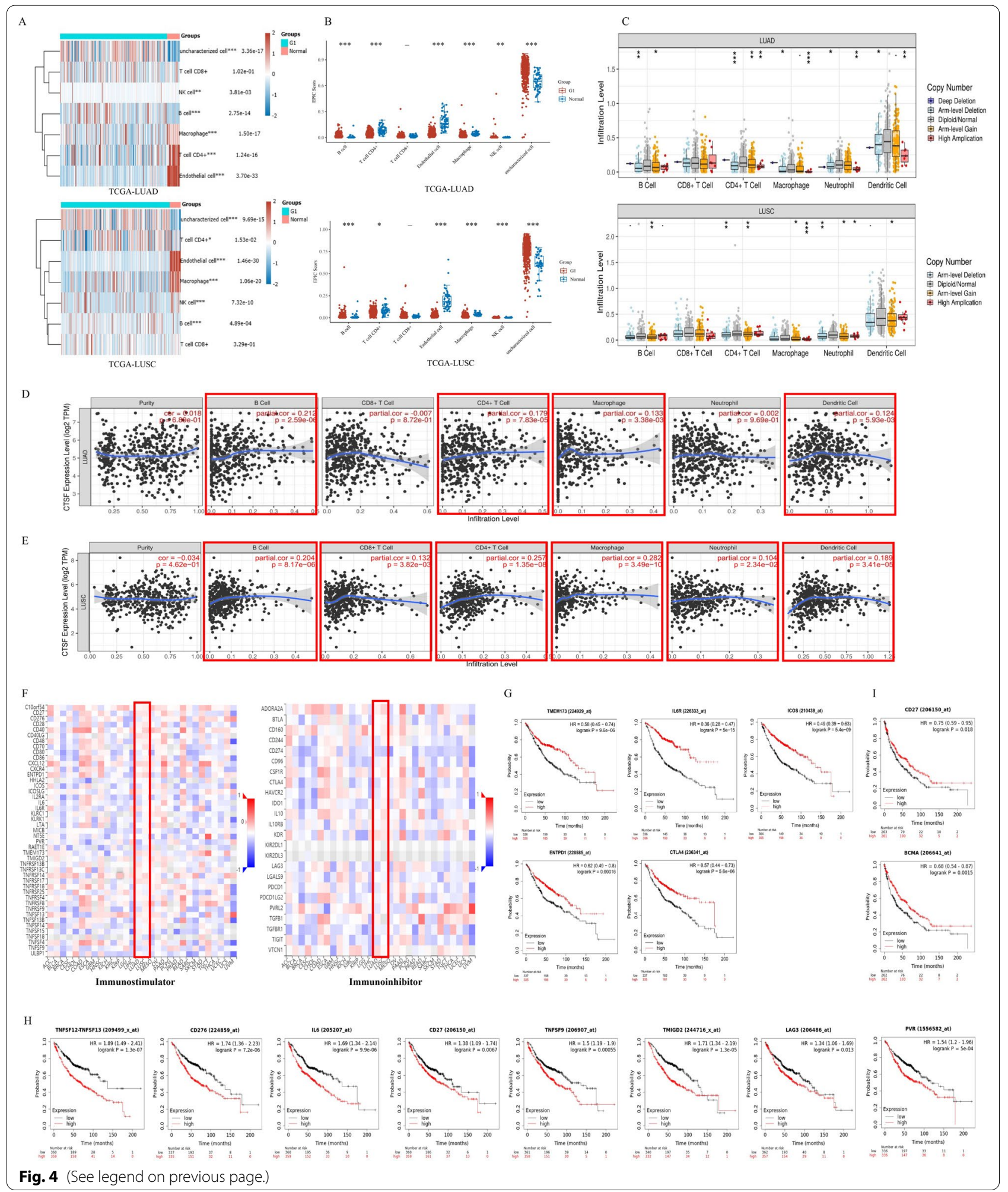

molecular markers of $\mathrm{CD} 8^{+} \mathrm{T}$ cells, general $\mathrm{T}$ cells, $\mathrm{B}$ cells, Monocytes, TAMs, M1 macrophages, M2 macrophages, Neutrophils, DCs, Th1 cells, Th2 cells, Th17 cells and Treg cells. Moreover, analysis of CTSF-related immunomodulators with prognosis of NSCLC patients further proved that CTSF affected prognosis of NSCLC 
Table 2 Correlation of CTSF expression with that of immunoinhibitors in LUAD and LUSC by TISIDB

\begin{tabular}{|c|c|c|c|c|}
\hline \multirow[t]{2}{*}{ Immunoinhibitor } & \multicolumn{2}{|l|}{ LUAD } & \multicolumn{2}{|l|}{ LUSC } \\
\hline & rho & $p$ & rho & $p$ \\
\hline ADORA2A & 0.058 & 0.188 & 0.174 & $* * *$ \\
\hline BTLA & 0.004 & 0.935 & 0.039 & 0.389 \\
\hline CD160 & -0.010 & 0.828 & 0.073 & 0.102 \\
\hline CD244 & -0.115 & $* *$ & 0.050 & 0.263 \\
\hline CD274 & -0.177 & $* * *$ & -0.208 & $* * *$ \\
\hline CD96 & -0.059 & 0.182 & 0.056 & 0.213 \\
\hline CSF1R & 0.010 & 0.827 & 0.086 & 0.054 \\
\hline CTLA4 & -0.140 & $* *$ & -0.011 & 0.800 \\
\hline HAVCR2 & -0.087 & $*$ & 0.033 & 0.463 \\
\hline IDO1 & -0.069 & 0.117 & 0.064 & 0.152 \\
\hline IL10 & -0.008 & 0.860 & 0.133 & $* *$ \\
\hline IL10RB & -0.026 & 0.561 & 0.080 & 0.074 \\
\hline KDR & 0.131 & $* *$ & 0.091 & * \\
\hline LAG3 & -0.163 & $* * *$ & -0.020 & 0.658 \\
\hline LGALS9 & -0.010 & 0.819 & -0.043 & 0.339 \\
\hline PDCD1 & -0.089 & * & 0.053 & 0.234 \\
\hline PDCD1LG2 & -0.052 & 0.239 & -0.016 & 0.724 \\
\hline PVRL2 & -0.102 & * & 0.031 & 0.485 \\
\hline TGFB1 & 0.063 & 0.150 & 0.000 & 0.999 \\
\hline TGFBR1 & -0.056 & 0.201 & -0.072 & 0.107 \\
\hline TIGIT & -0.151 & $* * *$ & -0.007 & 0.875 \\
\hline VTCN1 & 0.157 & $* * *$ & 0.032 & 0.468 \\
\hline
\end{tabular}

via regulating immune responses. Survival analysis displayed that high expression of four CTSF-related immunostimulators (TMEM173, IL6R, ICOS and ENTPD1) and one CTSF-related immunoinhibitor (CTLA-4) signified favorable prognosis of LUAD patients. In contrast, high expression of seven CTSF-related immunostimulators (TNFSF13, CD276, IL6, CD27, PVR, TNFSF9, TMIGD2) and one CTSF-related immunoinhibitor (LAG-3) were associated with poor prognosis of LUAD patients. For LUSC patients, high expression of two CTSF-related immunostimulators (CD27 and TNFRSF17) were related with favorable prognosis. CD27 was the only common CTSF-related immunostimulator associated with prognosis of LUAD and LUSC, although its role in LUAD and LUSC was distinctively different, demonstrating that though LUAD and LUSC belong to NSCLC, CTSF-related immunomodulators in the two subtypes of NSCLC are quite different.

Cancer immunotherapy encompasses a number of different treatments aimed at stimulating immune system in order to promote recognition and elimination of tumor cells [32]. In the past decade, immune checkpoints inhibitors (ICIs) have emerged as anticancer agents targeting inhibitory receptors (e.g. CTLA-4, PD-1, LAG-3, TIM-3)
Table 3 Correlation of CTSF expression with that of immunostimulators in LUAD and LUSC by TISIDB

\begin{tabular}{|c|c|c|c|c|}
\hline \multirow[t]{2}{*}{ Immunostimulators } & \multicolumn{2}{|l|}{ LUAD } & \multicolumn{2}{|l|}{ LUSC } \\
\hline & rho & $p$ & rho & $p$ \\
\hline C10orf54 & 0.040 & 0.362 & 0.069 & 0.124 \\
\hline CD27 & 0.102 & * & 0.103 & * \\
\hline CD276 & -0.088 & * & 0.045 & 0.312 \\
\hline CD28 & 0.052 & 0.237 & 0.100 & $*$ \\
\hline CD40 & 0.026 & 0.551 & 0.193 & $* * *$ \\
\hline CD40LG & 0.132 & $* *$ & 0.100 & $*$ \\
\hline CD48 & 0.050 & 0.259 & 0.063 & 0.160 \\
\hline CD70 & -0.023 & 0.595 & 0.135 & $* *$ \\
\hline CD80 & -0.086 & 0.050 & 0.009 & 0.842 \\
\hline CD86 & -0.063 & 0.150 & 0.053 & 0.234 \\
\hline CXCL12 & 0.167 & $* * *$ & 0.150 & $* * *$ \\
\hline CXCR4 & 0.016 & 0.712 & 0.077 & 0.084 \\
\hline ENTPD1 & 0.101 & * & 0.175 & $* * *$ \\
\hline HHLA2 & -0.017 & 0.698 & -0.027 & 0.541 \\
\hline ICOS & -0.120 & $* *$ & 0.010 & 0.831 \\
\hline ICOSLG & 0.106 & $*$ & 0.030 & 0.499 \\
\hline IL2RA & -0.137 & $* *$ & 0.003 & 0.941 \\
\hline IL6 & -0.160 & $* * *$ & -0.031 & 0.491 \\
\hline IL6R & 0.124 & $* *$ & 0.004 & 0.922 \\
\hline $\mathrm{KLRC1}$ & -0.181 & $* * *$ & 0.026 & 0.554 \\
\hline KLRK1 & -0.113 & $*$ & -0.027 & 0.546 \\
\hline LTA & -0.021 & 0.634 & 0.028 & 0.525 \\
\hline MICB & -0.165 & $* * *$ & 0.041 & 0.362 \\
\hline NT5E & 0.012 & 0.778 & -0.027 & 0.549 \\
\hline PVR & -0.263 & $* * *$ & -0.022 & 0.621 \\
\hline RAET1E & -0.175 & $* * *$ & -0.089 & $*$ \\
\hline TMEM173 & 0.158 & $* * *$ & 0.084 & 0.061 \\
\hline TMIGD2 & -0.104 & $*$ & 0.033 & 0.458 \\
\hline TNFRSF13B & 0.129 & $* *$ & 0.014 & 0.756 \\
\hline TNFRSF13C & 0.068 & 0.125 & -0.006 & 0.899 \\
\hline TNFRSF14 & 0.061 & 0.167 & 0.159 & $* * *$ \\
\hline TNFRSF17 & 0.126 & $* *$ & 0.103 & * \\
\hline TNFRSF 18 & -0.018 & 0.685 & -0.047 & 0.294 \\
\hline TNFRSF25 & 0.045 & 0.311 & -0.064 & 0.151 \\
\hline TNFRSF4 & 0.032 & 0.467 & 0.042 & 0.343 \\
\hline TNFRSF8 & -0.062 & 0.157 & 0.090 & $*$ \\
\hline TNFRSF9 & -0.141 & $* *$ & 0.065 & 0.147 \\
\hline TNFSF13 & 0.089 & $*$ & 0.045 & 0.320 \\
\hline TNFSF13B & -0.041 & 0.348 & 0.073 & 0.101 \\
\hline TNFSF14 & -0.039 & 0.377 & -0.019 & 0.669 \\
\hline TNFSF15 & 0.072 & 0.103 & 0.028 & 0.525 \\
\hline TNFSF18 & NA & NA & -0.154 & $* * *$ \\
\hline TNFSF4 & -0.090 & $*$ & 0.099 & $*$ \\
\hline TNFSF9 & -0.143 & $* *$ & -0.102 & $*$ \\
\hline ULBP1 & 0.029 & 0.511 & 0.067 & 0.632 \\
\hline
\end{tabular}


and ligands (PD-L1) expressed on T lymphocytes, antigen presenting cells and tumor cells and elicit an antitumor response by stimulating immune system and dramatically improved prognosis of many cancer patients including NSCLC patients [33]. Despite being traditionally considered as exhaustion T-cell markers [34, 35], PD-1, LAG-3 and TIM-3 are expressed preferentially in activated tumor infiltrating lymphocytes (TILs). Among these ICIs, CTLA-4 is a CD28 homolog with much higher binding affinity for $\mathrm{B} 7$ and was the first immune checkpoint targeted for cancer therapy in clinical practice $[36,37]$. Regretfully, the monodrug therapy that blocked CTLA-4 pathway failed in showed benefit in OS in NSCLC patients [38]. Our findings that high expression of CTSF-related CTLA-4 was associated with better OS of LUAD patients might explain the failure to a certain extent. The underlying mechanism is worth investigating.

LAG-3 (also CD223) is a 498-amino acid type I transmembrane protein with high structural homology with CD4 protein and capacity to bind MHC class II molecules $[39,40]$. LAG-3 molecule is expressed on CD4 ${ }^{+}$ and $\mathrm{CD}^{+} \mathrm{T}$ cells, Tregs, $\mathrm{B}$ cells and plasmacytoid dendritic cells. LAG-3 signaling plays a negative regulatory role in $\mathrm{T}$ helper 1 (Th1) cell activation, proliferation, and cytokine secretion. MHC-II is considered the canonical ligand of LAG-3 [41]. Currently, Relatlimab (BMS986016), the first commercially available monoclonal antibody directed against LAG-3, has been used in more than 20 clinical trials [42]. The trial NCT01968109 is evaluating the efficacy of Relatlimab as a monotherapy or in combination with Nivolumab (an anti-PD-1 antibody) in advanced solid tumors as well as NSCLC. PD-1 is expressed in T/B cells, NK, and MDSCs after their activation. In contrast to anti-CTLA-4 antibodies that fulfill their role in initial activation of $\mathrm{T}$ cells, main function of PD-1 is to limit activity of T cells in peripheral tissues [43]. Although the monodrug therapy that inhibited CTLA-4 pathway failed in improving OS in NSCLC patients [38], PD-1/PD-L1 (PD-1 ligand 1) checkpoint inhibitors have shown impressive results that have changed the landscape of NSCLC therapy [44-46]. Notably, association of elevated LAG-3 expression with insensitivity to PD-1 axis blockade suggested independence of these immune evasion pathways [47]. In the study, increased expression of CTSF-related LAG-3 with poor prognosis of LUAD patients while no association of CTSF-related PD-1/PD-L1 with prognosis of LUAD patients further demonstrated that the two immune evasion pathways are independent to each other. LAG-3 inhibitors might benefit LUAD patients independent to PD-1/PD-L1 expression.

CD27 (also TNFRSF7) is a member of the tumor necrosis factor receptor superfamily physiologically expressed on $\mathrm{CD}_{4}^{+}$and $\mathrm{CD}^{+} \mathrm{T}$ cells, NK cells and thymocytes [48, 49]. By binding to its natural ligand CD70, CD27 signaling enhances T-cell proliferation and differentiation to effector and memory T cells. After CD27 agonistic antibody varlilumab showed promising preclinical efficacy in haematological as well as solid cancers [50-52], varlilumab (also CDX-1127, 1F5), a human monoclonal antibody $(\mathrm{mAb})$ directed at $\mathrm{CD} 27$, has entered clinical trials both in haematological cancers and advanced solid tumors (NCT01460134 and NCT02335918) [53-55]. Presently, the clinical trial results are only available for colorectal and ovarian cancer showing that 10\% (5/49) of ovarian cancer patients achieved a partial response (PR) and 39\% (19/49) a stable disease (SD) [55]. Our findings that the increased expression of CTSF-related CD27 with poor prognosis of LUAD patients while related with favorable prognosis of LUSC suggested that CTSFrelated CD27 may play different roles in LUAD and LUSC and hinted that expression level of CTSF and subtype of NSCLC are the two key factors to predict the efficacy of CD27 monoclonal antibody for patients with NSCLC. CD27 monoclonal antibody might benefit patients with LUAD rather than LUSC.

TNFSF13 is a proliferation-inducing ligand playing an important role in B cell development [56]. The clinical significance of TNFSF13 in several cancers was previously analyzed such as NSCLC, breast cancer, leukemia, and other tumor types. In NSCLC, TNFSF13 is shown to be an independent prognostic factor in the 5-year overall survival rate [57]. In acute myeloid leukemia (AML), TNFSF13 is considered as a positive regulator of AML-initiating cells [58]. In triple-negative breast cancer (TNBC), the upregulated TNFSF13 is found to be correlated with a poor response to chemotherapy, suggesting that TNFSF13 could be a predictive biomarker for patients receiving chemotherapy [59]. In the study, CTSF-related TNFSF13 was shown to be related with poor prognosis of LUAD patients. The value of monoclonal antibody targeting TNFSF13 are worth exploring in clinic.

Human cysteine proteinases include more than 10 members [8]. Among these CTSs, several cysteine proteinases have been reported to be associated with progression of cancers by regulating immune response although their functions are entirely different in different cancers. For example, elevated CTSB is associated with increased immune cell infiltration of tumor-associated $B$ cells and mast cells, and facilitates progression and metastasis of PymT-induced mammary carcinomas [60]. CTSS regulates antigen processing and CD4 and CD8 T cell-mediated immune responses. Loss of CTSS activity reduces lymphoma growth by limiting communication with $\mathrm{CD} 4 \mathrm{~T}$ follicular helper cells while inducing antigen 
diversification and activation of CD8 T cells [61]. In colorectal cancer, CTSL is reported to be one of the genes involved in immunosuppression [62]. Increased levels of enzymatically active CTSC contributes to squamous cell carcinoma growth via regulating infiltrating immune cells in neoplastic skin, development of angiogenic vasculature, and squamous cell carcinoma growth [63]. In Endometrial cancer, CTSW is found to be one of the genes correlated positively with tumor infiltration levels of B cells, $\mathrm{CD}^{+} \mathrm{T}$ cells, CD4 ${ }^{+} \mathrm{T}$ cells, macrophages, and dendritic cells, indicating that composition of tumor microenvironment affects clinical outcomes of Endometrial cancer patients, and suggesting that it may provide a basis for development of novel prognostic biomarkers and immunotherapies for patients with Endometrial cancer [64]. Presently, CTSF has been shown to likely play vital roles in immune responses via regulating MHC II [17-19]. Thus, our findings that CTSF and CTSF-related immunomodulators were associated with prognosis of NSCLC strongly suggested that CTSF might play an important role in immune response of NSCLC. Classification of immune cells and immunomodulators based on CTSF expression will help to screen the clinically applicable individuals for immunotherapy. Therefore, targeting CTSF might hew out novel therapeutics of NSCLC by regulating immune responses.

Despite some merits of the current study, there were several limitations. First, because all of the points were speculated from the public databases online available using the bioinformatic methods, the experiments in vitro and in vivo are required to validate the relationship between CTSF and immune responses and explore the mechanisms underpinning CTSF-medicated tumor immunity of NSCLC. Second, the appropriate clinical samples are needed to prove the prognostic impact of CTSF and association between CTSF and immune responses in NSCLC. Third, the agonist or recombinant CTSF need to be explored and tested in animal models to provide novel ways for improving the precision immunotherapy of NSCLC in the future.

\section{Conclusions}

Taken together, the study reported for the first time that CTSF may influence prognosis of NSCLC patients via regulating immune responses and might be a novel therapeutic target of NSCLC.

\footnotetext{
Abbreviations

CTSF: Cathepsin F; LUAD: Lung adenocarcinoma; LUSC: Lung squamous cell carcinoma; NSCLC: Non-small cell lung cancer; TCGA: The cancer genome atlas; TIMER: The tumor immune estimation resource; MHC: Major histocompatibility complex; GTE: Genotype-tissue expression; CCLE: Cancer cell line encyclopedia; IHC: Immunohistochemistry; THPA: The human protein atlas; OS: Overall survival; FPS: First-progression survival; PPS: Post-progression
}

survival; HR: Hazard ratio; Cls: Confidence intervals; TMB: Tumor mutational burden; MSI: Microsatellite instability; NGS: Next-generation sequencing; ENCORI: Encyclopedia of RNA interactomes; GO: Gene ontology; KEGG: Kyoto encyclopedia of genes and genomes; PPI: Protein-protein interaction; DCM: Dilated cardiomyopathy; TNBC: Triple-negative breast cancer; AML: Acute myeloid leukemia; ALK: Anaplastic lymphoma kinase; EGFR: Epidermal growth factor receptor.

\section{Supplementary Information}

The online version contains supplementary material available at https://doi. org/10.1186/s12890-021-01796-w.

Additional file 1. Table S1. Expression of CTSF in human cancer cell lines. Additional file 2. Figure S1. (A, B) Co-expressed genes with CTSF identified in LUAD $(\mathbf{A})$ and LUSC (B), respectively (Red and green dots represented the positively and negatively correlated genes with CTSF, respectively).

Acknowledgements

Not applicable.

\section{Authors' contributions}

The author contributions were as follows: FYJ and LYS, concept and design; LYS, XHW, WC, YW, ML, SYZ, HX, HL, RZL, XHL, XJT, and HZZ collection of the data available online and analysis; LYS, FYJ, and XHL, writing of the paper. All the authors read and approved the final manuscript.

\section{Funding}

This work was supported by grants from the Cultivating Project for Young Scholar at Hubei University of Medicine (No: 2018QDJZR01) awarded to Dr. Fuyun Ji. The funders had no role in study design, in collection, analysis and interpretation of data, in writing of the manuscript, and in decision to submit the article for publication.

\section{Availability of data and materials}

The datasets used and/or analyzed during the current study are available from the corresponding author on reasonable request.

\section{Declarations}

Ethics approval and consent to participate

Not applicable.

\section{Consent for publication}

Not applicable.

\section{Competing interests}

The authors declare that they have no competing interests.

\section{Author details}

${ }^{1}$ Department of Medical Biology, School of Basic Medical Science, Hubei University of Medicine, Shiyan 442000, Hubei Province, China. ${ }^{2}$ Institute of Biomedical Research, Hubei University of Medicine, Shiyan 442000, Hubei Province, China. ${ }^{3}$ Taihe Hospital, Hubei University of Medicine, Shiyan 442000, Hubei Province, China. ${ }^{4}$ School of Clinical Medicine, Shandong First Medical University, Jinan 250000, Shandong Province, China. ${ }^{5}$ Department of Pathology, School of Basic Medical Science, Hubei University of Medicine, Shiyan 442000, Hubei Province, China. ${ }^{6}$ Hubei Key Laboratory of Embryonic Stem Cell Research, School of Basic Medical Science, Hubei University of Medicine,

Shiyan 442000, Hubei Province, China.

Received: 22 September 2021 Accepted: 14 December 2021

Published online: 20 December 2021 


\section{References}

1. Sung H, Ferlay J, Siegel RL, et al. Global cancer statistics 2020: GLOBOCAN estimates of incidence and mortality worldwide for 36 cancers in 185 countries. CA Cancer J Clin. 2021. https://doi.org/10.3322/caac.21660.

2. Didkowska J, Wojciechowska U, Mańczuk M, Łobaszewski J. Lung cance epidemiology: contemporary and future challenges worldwide. Ann Transl Med. 2016:4:150

3. Rahal Z, El Nemr S, Sinjab A, Chami H, Tfayli A, Kadara H. Smoking and lung cancer: a geo-regional perspective. Front Oncol. 2017:7:194.

4. Hirsch FR, Scagliotti GV, Mulshine JL, Kwon R, Curran WJ, Wu YL, Paz-Ares L. Lung cancer: current therapies and new targeted treatments. Lancet. 2017;389:299-311.

5. Huang MY, Jiang XM, Wang BL, Sun Y, Lu JJ. Combination therapy with PD-1/PD-L1 blockade in non-small cell lung cancer: strategies and mechanisms. Pharmacol Ther. 2021;219:107694.

6. De Carlo E, Schiappacassi M, Del Conte A, Stanzione B, Bearz A. Combination of chemotherapy and ALK Inhibitors in ALK-positive NSCLC. J Thorac Oncol. 2021;16(5):e31-2.

7. Aredo JV, Mambetsariev I, Hellyer JA, Amini A, Neal JW, Padda SK, et al. Durvalumab for Stage III EGFR-Mutated NSCLC After Definitive Chemoradiotherapy. J Thorac Oncol. 2021. https://doi.org/10.1016/j.jtho.2021.01. 1628.

8. Bond JS, Butler PE. Intracellular proteases. Annu Rev Biochem. 1987;56:333-64

9. Chapman HA, Riese RJ, Shi GP. Emerging roles for cysteine proteases in human biology. Annu Rev Physiol. 1997;59:63-88.

10. Rawlings ND, Barrett AJ. Families of cysteine peptidases. Methods Enzymol. 1994;244:461-86

11. Bühling F, Fengler A, Brandt W, Welte T, Ansorge S, Nägler DK. Review: novel cysteine proteases of the papain family. Adv Exp Med Biol. 2000:477:241-54

12. Santamaría I, Velasco G, Pendás AM, Paz A, López-Otín C. Molecular cloning and structural and functional characterization of human cathepsin $\mathrm{F}$ a new cysteine proteinase of the papain family with a long propeptide domain. J Biol Chem. 1999;274(20):13800-9.

13. Arora K, Herroon M, Al-Afyouni MH, Toupin NP, Rohrabaugh TN, Loftus LM, et al. Catch and release photosensitizers: combining dual-action ruthenium complexes with protease inactivation for targeting invasive cancers. Am Chem Soc. 2018;140(43):14367-80

14. Zhang C, Zhang M, Song S. Cathepsin D enhances breast cancer invasion and metastasis through promoting hepsin ubiquitin-proteasome degradation. Cancer Lett. 2018:438:105-15.

15. Frugé AD, Smith KS, Bail JR, Rais-Bahrami S, Demark-Wahnefried W. Biomarkers associated with tumor Ki67 and cathepsin L gene expression in prostate cancer patients participating in a presurgical weight loss trial. Front Oncol. 2020;10:544201.

16. Bararia D, Hildebrand JA, Stolz S, Haebe S, Alig S, Trevisani CP, et al. Cathepsin $\mathrm{S}$ alterations induce a tumor-promoting immune microenvironment in follicular lymphoma. Cell Rep. 2020;31(5):107522.

17. Shi GP, Bryant RA, Riese R, Verhelst S, Driessen C, Li Z, et al. Role for cathepsin $\mathrm{F}$ in invariant chain processing and major histocompatibility complex class II peptide loading by macrophages. J Expt Med. 2000;191:1177-86.

18. Somoza JR, Palmer JT. Ho JDJ The crystal structure of human cathepsin F and its implications for the development of novel immunomodulators. Mol Biol. 2002;322(3):559-68.

19. Boussiotis V. Somatic mutations and immunotherapy outcome with CTLA-4 blockade in melanoma. N Engl J Med. 2014;371(23):30-2.

20. Chandrashekar DS, Bashel B, Balasubramanya SAH, Creighton CJ, Rodriguez IP, Chakravarthi BVSK, et al. UALCAN: A portal for facilitating tumor subgroup gene expression and survival analyses. Neoplasia. 2017;19(8):649-58.

21. Broglio KR, Berry DA. Detecting an overall survival benefit that is derived from progression-free survival. J Natl Cancer Inst. 2009;101:1642-9.

22. Hotta K, Kiura K, Fujiwara Y, Takigawa N, Hisamoto A, Ichihara E, et al. Role of survival post-progression in phase III trials of systemic chemotherapy in advanced non-small-cell lung cancer: a systematic review. PLOS ONE. 2011;6:e26646

23. Gyorffy B, Surowiak P, Budczies J, Lanczky A. Online survival analysis software to assess the prognostic value of biomarkers using transcriptomic data in non-small-cell lung cancer. PLoS ONE. 2013;8(12):e82241.
24. Vasaikar S, Straub P, Wang J, Zhang B. LinkedOmics: analyzing multiomics data within and across 32 cancer types. Nucleic Acids Res. 2018:46(D1):D956-63.

25. Ji C, Zhao Y, Kou YW, Shao H, Guo L, Bao CH, et al. Cathepsin F knockdown induces proliferation and inhibits apoptosis in gastric cancer cells. Oncol Res. 2018;26(1):83-93.

26. Zheng L, Cao J, Liu L, Xu H, Chen L, Kang L, et al. Long noncoding RNA LINC00982 upregulates CTSF expression to inhibit gastric cancer progression via the transcription factor HEY1. Am J Physiol Gastrointest Liver Physiol. 2021;320(5):G816-28.

27. Di Rosa M, Sanfilippo C, Libra M, Musumeci G, Malaguarnera L. Different pediatric brain tumors are associated with different gene expression profiling. Acta Histochem. 2015;117(4-5):477-85.

28. Janic A, Valente LJ, Wakefield MJ, Di Stefano L, Milla L, et al. DNA repair processes are critical mediators of p53-dependent tumor suppression. Nat Med. 2018;24(7):947-53.

29. Oliveras-Ferraros C, Vazquez-Martin A, Cuyàs E, Corominas-Faja B, Rodríguez-Gallego E, Fernández-Arroyo $S$, et al. Acquired resistance to metformin in breast cancer cells triggers transcriptome reprogramming toward a degradome-related metastatic stem-like profile. Cell Cycle. 2014;13(7):1132-44.

30. Busch R, Kollnberger S, Mellins ED. HLA associations in inflammatory arthritis: emerging mechanisms and clinical implications. Nat Rev Rheumatol. 2019;15(6):364-81.

31. Schröder B. The multifaceted roles of the invariant chain CD74-More than just a chaperone. Biochim Biophys Acta. 2016;1863:1269-81.

32. Yang Y. Cancer immunotherapy: harnessing the immune system to battle cancer. J Clin Invest. 2015;125:3335-7.

33. Das $\mathrm{S}$, Johnson DB. Immune-related adverse events and anti-tumor efficacy of immune checkpoint inhibitors. J Immunother Cancer. 2019:7:306.

34. Giordano M, Henin C, Maurizio J, Imbratta C, Bourdely P, Buferne M, et al. Molecular profiling of CD8 T cells in autochthonous melanoma identifies Maf as driver of exhaustion. EMBO J. 2015;34:2042-58.

35. Wherry EJ, Ha S-J, Kaech SM, Haining WN, Sarkar S, Kalia V, et al. Molecular signature of CD8 + T cell exhaustion during chronic viral infection. Immunity. 2007;27:670-84.

36. Postow MA, Callahan MK, Wolchok JD. Immune checkpoint blockade in cancer therapy. J Clin Oncol. 2015;33:1974-82.

37. Zatloukal P, Heo DS, Park K, et al. Randomized phase II clinical trial comparing tremelimumab (CP-675,206) with best supportive care (BSC) following first-line platinum-based therapy in patients (pts) with advanced non-small cell lung cancer (NSCLC). J Clin Oncol (Meeting Abstracts). 2009;27(15S):8071.

38. Gao C, Fu Q, Su B, Song H, Zhou S, Tan F, et al. The involvement of cathepsin F gene (CTSF) in turbot (Scophthalmus maximus L.) mucosal immunity. Fish Shellfish Immunol. 2017;66:270-9.

39. Woo S-R, Turnis ME, Goldberg MV, Bankoti J, Selby M, Nirschl CJ, et al. Immune inhibitory molecules LAG-3 and PD-1 synergistically regulate T-cell function to promote tumoral immune escape. Cancer Res. 2012;72:917-27.

40. Andrews LP, Marciscano AE, Drake CG, Vignali DAA. LAG3 (CD223) as a cancer immunotherapy target. Immunol Rev. 2017;276:80-96.

41. Qin S, Xu L, Yi M, Yu S, Wu K, Luo S. Novel immune checkpoint targets: moving beyond PD-1 and CTLA-4. Mol Cancer. 2019;18:155.

42. Yu X, Huang X, Chen X, Liu J, Wu C, Pu Q, et al. Characterization of a novel anti-human lymphocyte activation gene 3 (LAG-3) antibody for cancer immunotherapy. MAbs. 2019;11:1139-48.

43. Nguyen L, Ohashi P. Clinical blockade of PD1 and LAG3- Potential mechanisms of action. Nat Rev Immunol. 2015:15:45-56.

44. Borghaei H, Paz-Ares L, Horn L, Spigel DR, Steins M, Ready NE, et al. Nivolumab versus docetaxel in advanced nonsquamous non-small-cell lung cancer. N Engl J Med. 2015;373(17):1627-39.

45. Brahmer J, Reckamp KL, Baas P, Crinò L, Eberhardt WE, Poddubskaya E, et al. Nivolumab versus docetaxel in advanced squamous-cell non-smallcell lung cancer. N Engl J Med. 2015;373(2):123-35.

46. Rizvi NA, Mazières J, Planchard D, Stinchcombe TE, Dy GK, Antonia SJ, et al. Activity and safety of nivolumab, an anti-PD-1 immune checkpoint inhibitor, for patients with advanced, refractory squamous non-small-cell lung cancer (Checkmate 063): a phase 2, single-arm trial. Lancet Oncol. 2015;16(3):257-65. 
47. Datar I, Sanmamed MF, Wang J, Henick BS, Choi J, Badri T, et al. Expression analysis and significance of PD-1, LAG-3, and TIM-3 in human non-small cell lung cancer using spatially resolved and multiparametric single-cell analysis. Clin Cancer Res. 2019;25(15):4663-73.

48. Borst J, Hendriks J, Xiao Y. CD27 and CD70 in T cell and B cell activation. Curr Opin Immunol. 2005;17:275-81.

49. Bodmer IL, Schneider P, Tschopp J. The molecular architecture of the TNF superfamily. Trends Biochem Sci. 2002;27:19-26.

50. Vitale LA, He L-Z, Thomas LJ, Widger J, Weidlick J, Crocker A, et al. Development of a human monoclonal antibody for potential therapy of CD27expressing lymphoma and leukemia. Clin Cancer Res. 2012;18:3812-21.

51. He LZ, Prostak N, Thomas LJ, Vitale L, Weidlick J, Crocker A, et al. Agonist anti-human CD27 monoclonal antibody induces $T$ cell activation and tumor immunity in human CD27-transgenic mice. J Immunol. 2013;191:4174-83.

52. Ramakrishna V, Sundarapandiyan K, Zhao B, Bylesjo M, Marsh HC, Keler T. Characterization of the human T cell response to in vitro CD27 costimulation with varlilumab. J Immunother Cancer. 2015;3:37.

53. Burris HA, Infante JR, Ansell SM, Nemunaitis JJ, Weiss GR, Villalobos VM. Safety and activity of Varlilumab, a novel and first-in-class agonist antiCD27 antibody, in patients with advanced solid tumors. J Clin Oncol. 2017:35:2028-36.

54. Ansell SM, Northfelt DW, Flinn I, et al. Phase I evaluation of an agonist anti-CD27 human antibody (CDX-1127) in patients with advanced hematologic malignancies. JCO. 2014;32:3024.

55. Sanborn RE, Pishvaian MJ, Callahan MK, et al. Anti-CD27 agonist antibody varlilumab (varli) with nivolumab (nivo) for colorectal (CRC) and ovarian (ova) cancer: phase (Ph) 1/2 clinical trial results. J Clin Oncol. 2018;36:3001.

56. Bossen C, Schneider P. BAFF, APRIL and their receptors: structure, function and signaling. Semin Immunol. 2006;18(5):263-75.

57. Qian Z, Qingshan C, Chun J, Huijun Z, Feng L, Qiang W, et al. High expression of TNFSF13 in tumor cells and fibroblasts is associated with poor prognosis in non-small cell lung cancer. Am J Clin Pathol. 2014;141(2):226-33.

58. Chapellier M, Peña-Martínez P, Ramakrishnan R, Eriksson M, Talkhoncheh MS, Orsmark-Pietras $C$, et al. Arrayed molecular barcoding identifies TNFSF13 as a positive regulator of acute myeloid leukemia-initiating cells. Haematologica. 2019;104(10):2006-16.

59. Lin HY, Kuei CH, Lee HH, Lin CH, Chen YL, Chen CL, et al. TNFSF13 upregulation confers chemotherapeutic resistance via triggering autophagy initiation in triple-negative breast cancer. J Mol Med (Berl). 2020;98(9):1255-67.

60. Sevenich L, Werner F, Gajda M, Schurigt U, Sieber C, Müller S, et al. Transgenic expression of human cathepsin B promotes progression and metastasis of polyoma-middle-T-induced breast cancer in mice. Oncogene. 2011;30(1):54-64.

61. Dheilly E, Battistello E, Katanayeva N, Sungalee S, Michaux J, Duns G, et al. Cathepsin $\mathrm{S}$ regulates antigen processing and $\mathrm{T}$ cell activity in nonhodgkin lymphoma. Cancer Cell. 2020;37(5):674-89.

62. Ohno Y, Kitamura H, Takahashi N, Ohtake J, Kaneumi S, Sumida K, et al. IL-6 down-regulates HLA class II expression and IL-12 production of human dendritic cells to impair activation of antigen-specific CD4( $\left.{ }^{+}\right) \mathrm{T}$ cells. Cancer Immunol Immunother. 2016;65(2):193-204.

63. Ruffell B, Affara NI, Cottone L, Junankar S, Johansson M, DeNardo DG, et al. Cathepsin $\mathrm{C}$ is a tissue-specific regulator of squamous carcinogenesis. Genes Dev. 2013;27(19):2086-98.

64. Brown J, Matutes E, Singleton A, Price C, Molgaard H, Buttle D, et al. Lymphopain, a cytotoxic T and natural killer cell-associated cysteine proteinase. Leukemia. 1998;12(11):17.

\section{Publisher's Note}

Springer Nature remains neutral with regard to jurisdictional claims in published maps and institutional affiliations. 